\title{
Antibiofilm agents with therapeutic potential against enteroaggregative Escherichia coli
}

\section{David A. Kwasi ${ }^{1,2}$, Chinedum P. Babalola ${ }^{2,3}$, Olujide O. Olubiyi ${ }^{4,5}$, Jennifer Hoffmann, Ikemefuna C. Uzochukwu ${ }^{6}$, and Iruka N. Okeke ${ }^{1 *}$.}

1. Department of Pharmaceutical Microbiology, Faculty of Pharmacy, University of Ibadan, Oyo State, Nigeria.

2. Department of Pharmaceutical Chemistry, Faculty of Pharmacy, University of Ibadan, Ibadan, Oyo State, Nigeria.

3. Center for Drug Discovery, Development and Production, Faculty of Pharmacy, University of Ibadan, Oyo State, Nigeria.

4. Department of Pharmaceutical Chemistry, Faculty of Pharmacy, Obafemi Awolowo University, Ile-Ife, Osun State, Nigeria.

5. Institute of Drug Research and Development, Afe Babalola University, Ado-Ekiti, Nigeria 6. Department of Pharmaceutical and Medicinal Chemistry, Faculty of Pharmaceutical Sciences, Nnamdi Azikiwe University Awka, Anambra State, Nigeria.

*Corresponding author: Iruka N. Okeke. Department of Pharmaceutical Microbiology, Faculty of Pharmacy, University of Ibadan, Oyo State, Nigeria. +234 8053281714. iruka.n.okeke@gmail.com 


\section{Abstract}

\section{Background}

53 Enteroaggregative Escherichia coli (EAEC) is a predominant but neglected enteric

54 pathogen implicated in infantile diarrhoea and nutrient malabsorption. There are no non-

55 antibiotic approaches to dealing with persistent infection by these exceptional colonizers,

56 which form copious biofilms. We screened the Medicines for Malaria Venture Pathogen Box

57 for chemical entities that inhibit EAEC biofilm formation.

\section{$58 \quad$ Methodology}

59 We used two EAEC strains, 042 and MND005E, in a medium-throughput crystal violet-based

60 antibiofilm screen. Hits were confirmed in concentration-dependence, growth kinetic and

61 time course assays and activity spectra were determined against a panel of genome-sequenced

62 EAEC. Antibiofilm activity against isogenic EAEC mutants, molecular docking simulations

63 and comparative genomic analysis were used to identify the mechanism of action of one hit.

\section{Principal findings}

65 In all, five compounds (1.25\%) reproducibly inhibited biofilm accumulation by at least one

66 strain by $30-85 \%$ while inhibiting growth by under $10 \%$. Hits exhibited at least 10 -fold

67 greater antibiofilm activity than nitazoxanide, the only known EAEC biofilm inhibitor.

68 Reflective of known EAEC heterogeneity, only one hit was active against both screen

69 isolates, but three hits showed broad antibiofilm activity against a larger panel of strains.

70 Mechanism of action studies point to the EAEC anti-aggregation protein (Aap), dispersin, as

71 the target of compound MMV687800.

\section{Conclusions}

73 This study identified five compounds not previously described as anti-adhesins or Gram-

74 negative antibacterials with significant and specific EAEC antibiofilm activity. One

75 molecule, MMV687800, targets the EAEC Aap. In vitro small-molecule inhibition of EAEC 
76 colonization opens a way to new therapeutic approaches to preventing and treating EAEC

77 infection.

78

79 Keywords: Biofilm, biofilm inhibitors, diarrhoea Enteroaggregative Escherichia coli, 80 Pathogen Box. 


\section{Author summary}

82 Diarrhoea accounts for over half a million deaths in children under five annually. It

83 additionally contributes to childhood malnutrition as well as growth and development

84 deficiencies, particularly in low-income countries. Enteroaggregative Escherichia coli

85 (EAEC) causes diarrhoea that is often persistent and can also contribute to growth

86 deficiencies in young children. EAEC is a neglected pathogen that is often resistant to

87 antimicrobial drugs. Small molecules that block EAEC colonization may hold the key to

88 interfering with EAEC disease without promoting antimicrobial resistance. We screened the

89 Medicines for Malaria Ventures Pathogen Box for chemicals that can interfere with EAEC

90 biofilm formation, a key colonization indicator. Our screen identified five biofilm-inhibiting

91 molecules that did not interfere with bacterial viability and therefore are unlikely to exert

92 strong pressure for resistance. Molecular biology and computational investigations point to

93 the EAEC anti-aggregative protein, also known as dispersin, as a possible target for one of

94 these hit molecules. Optimizing EAEC antibiofilm hits will create templates that can be

95 employed for resolving EAEC diarrhoea and related infections. 


\section{Introduction}

103 Diarrhoea constitutes a huge global disease burden [1,2], accounting for approximately

104500,000 deaths among under-fives annually [3, 4]. Diarrhoea also contributes significantly to 105 malnutrition as well as growth and development shortfalls, particularly in low-income 106 countries [5,6]. The burden from diarrhoea is highest in Africa with Nigeria topping the list 107 on the Africa continent and ranking second only to India's contribution to the global burden 108 from the syndrome [4].

109 Infectious diarrhoea can be caused by a wide range of micro-organisms including, but in no 110 way limited to rotavirus, astrovirus, norovirus, Entamoeba, Cryptosporidium, multiple 111 subtypes of diarrhoeagenic Escherichia coli and Salmonella [7]. Enteroaggregative 112 Escherichia coli (EAEC) is a diarrhoeagenic E. coli subtype known to cause both acute and 113 persistent diarrhoea (the latter continuing for more than 14 days) [8]. EAEC strains are 114 additionally implicated in traveler's diarrhoea and foodborne outbreaks worldwide [9-11].

115 EAEC are epidemiologically important globally and are repeatedly detected at high 116 prevalence in many epidemiological studies [12-14], including our earlier and ongoing 117 research in West Africa [15-18]. The burden of EAEC infections and their impact on child 118 health necessitates a clear understanding of the pathogenesis of the disease, as well as 119 effective interventions. However, EAEC research is neglected even more than research on 120 other high-burden bacterial diarrhoeal pathogens such as rotavirus, enterotoxigenic E. coli 121 and Shigella $[19,20]$.

122 Hallmarks of EAEC infection include copious adherence to epithelial cells in a striking 123 'stacked brick' or 'aggregative' fashion as well as the formation of voluminous biofilms $124[16,21,22]$. Biofilms are a complex community of organisms encased in an extracellular 125 matrix and EAEC in vitro and in vivo biofilms are believed to contribute to persistent 126 colonization and transmission of diarrhoea [23-25]. 
127 EAEC are genetically heterogeneous and difficult to delineate from commensals [20, 26, 27].

128 Although the molecular epidemiology of EAEC infection remains unclear, most strains

129 colonize the intestinal mucosa via the aggregative adherence fimbriae (AAFs) and other non-

130 structural adhesins [25,28], which also contribute to copious biofilm formation and

131 subsequent host pathogenesis [23,27,29]. These critical components of the EAEC pathogenic

132 cascade suggest that molecules which can interfere with or inhibit the assembly or function of

133 adhesins central to EAEC virulence could be promising therapeutic candidates.

134 We hypothesized that small molecules can interfere with or inhibit EAEC adherence to host

135 cells either by structurally modifying adhesins or competing for their receptor sites without

136 inhibiting growth. We tested our hypothesis by screening the Medicines for Malaria

137 Ventures' (MMV) Pathogen Box chemical library (https://www.mmv.org/mmv-

138 open/pathogen-box/about-pathogen-box), deploying a commonly used biofilm assay protocol

139 [28,30-32], adapted to medium-throughput. Pathogen Box is a curated compound library

140 containing 400 synthetic compounds arrayed in a 96-well format. Information about activities

141 of the compounds against Plasmodium falciparium and some neglected human pathogens is

142 provided with the library, but the compounds have not been tested against bacterial causes of

143 diarrhoea or other Gram-negative bacteria. The structures, physicochemical properties as well

144 as preliminary profiles of the compounds are provided with the box, providing useful insights

145 and good chemical starting points for neglected pathogen drug research.

147 Methods

148 Bacteria strains, plasmids, and culture conditions

149 Two EAEC strains, 042, a prototypical EAEC strain originally isolated in Peru [33,34] and

150 MND005E, an EAEC strain isolated from an ongoing case-control study in our laboratory in

151 Nigeria [18], were used for the preliminary screens and confirmatory assays. These strains

152 and 25 other biofilm-forming EAEC strains (Table 1) are isolates from diarrhoea patients 
153 under the age of five. Isogenic 042 mutants and laboratory strain ER2523 (NEB express)

154 shown in Table 1 were used to obtain preliminary information on mode of action of hits.

155 Molecular microbiology methods, comparative genomics and molecular docking were used

156 to confirm hit mechanism of action. Strains were routinely cultured in Luria Bertani (LB)

157 broth (Sigma Aldrich: Cat no. L3522), adding ampicillin (100 $\mu \mathrm{g} / \mathrm{ml})$ where necessary.

158 Strains were archived at $-80^{\circ} \mathrm{C}$ in sterile cryogenic vials in Luria Bertani broth (Mueller)

159 glycerol in ratio 1:1. Plasmids used or generated in the study are listed in Table 1.

\section{Chemical library}

162 Pathogen Box, a chemical library of 400 diverse, drug-like molecules, was a kind gift from

163 the Medicines for Malaria Venture (MMV, Switzerland). Compounds were supplied at 10

$164 \mathrm{mM}$ dissolved in $10 \mu \mathrm{l}$ dimethyl sulfoxide (DMSO) in sterile 96-well polystyrene plates (A-

165 E, 80 compounds per plate). Tentative hits were resupplied compounds from MMV and/or

166 purchased from Sigma Aldrich for downstream experiments. These were received as

167 powders then reconstituted in our laboratory in DMSO (Sigma Aldrich: Cat no. D5879). In

168 every set up, final concentration of DMSO did not exceed $1 \%$, which we confirmed in

169 preliminary assays had no effect on EAEC growth and biofilms. Stock solutions were stored

170 at $-20^{\circ} \mathrm{C}$, thawed, mixed and then diluted to required concentrations prior to each

171 experimental set up.

173 Biofilm inhibition assays

174 The medium throughput screen was set up in sterile 96-well flat bottom polystyrene plates

175 (Nunc: 260860). For each assay, $5 \mu \mathrm{l}$ of $200 \mu \mathrm{M}$ drug solutions dissolved in net DMSO

176 (Sigma Aldrich: Cat no. D5879) were pipetted into the 96-well plate using a multichannel

177 pipette (Gilson). Assay plates subsequently received $195 \mu \mathrm{l}$ of high glucose Dulbecco's

178 Modified Eagles Medium (DMEM, ThermoFisher Scientific, cat no. 11965092) containing 
179 overnight culture in ratio $1: 100$ to achieve a final drug concentration of $5 \mu \mathrm{M}$ in each test

180 well. Control wells received $2 \mu \mathrm{l}$ of DMSO vehicle and $198 \mu$ l of the same mixture of

181 DMEM and overnight culture to ensure a final DMSO concentration of $1 \%$ in control wells

182 [35]. Assay was set up in triplicate for control and each compound then incubated at $37^{\circ} \mathrm{C}$ for

$1838 \mathrm{~h}$. Planktonic cell growth was determined by quantifying optical densities at $595 \mathrm{~nm}$ using a

184 microplate spectrophotometer (Thermo scientific multiscan FC: Cat no. 51119000). Plates

185 were thoroughly washed three times with $200 \mu \mathrm{l}$ of sterile PBS per well using a microplate

186 washer (Micro wash 1100 from Global diagnostics) then air-dried and fixed with 75\%

187 ethanol for 10 minutes. After the plates were dried, we stained with $0.5 \%$ crystal violet for 5

188 minutes then washed thoroughly with water. We dried plates completely then eluted crystal

189 violet using $200 \mu \mathrm{l}$ of $95 \%$ ethanol for 20 minutes. Biofilm was quantified by determining

190 optical density of eluted crystal violet at $570 \mathrm{~nm}$ using a multiscan microplate

191 spectrophotometer.

192 Antibiofilm and growth inhibitory effects of each compound were computed from the

193 averages of 3 replicates applying the following formulae:

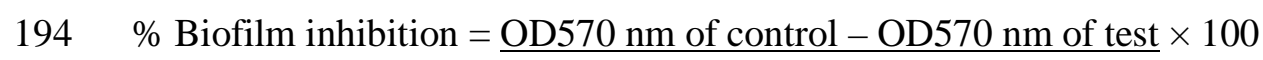

OD570 nm of control

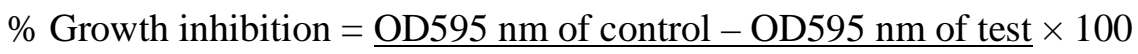

OD595 nm of control

198 Antibiofilm activity of compounds identified as initial hits was additionally confirmed in 199 concentration dependent assays set up in sterile flat bottom 96-well polystyrene plate.

200 Concentrations of compounds were prepared in serial 2-fold dilutions ranging from 0.3125

$201 \mu \mathrm{M}$ to $20 \mu \mathrm{M}$. Serially diluted compounds were added in triplicate to $195 \mu \mathrm{L}$ of DMEM

202 containing standardized overnight culture of organism. For retesting of previously described

203 antibiofilm agent nitoxazanide (NTZ); $15 \mu \mathrm{g} / \mathrm{mL}(48.8 \mu \mathrm{M}), 20 \mu \mathrm{g} / \mathrm{mL}(65.15 \mu \mathrm{M})$ and 25 
$204 \mu \mathrm{g} / \mathrm{mL}(81.45 \mu \mathrm{M})$ earlier reported to exhibit significant biofilm inhibition in EAEC [28]

205 were tested.

206

207 Plates were incubated at $37^{\circ} \mathrm{C}$ for $8 \mathrm{~h}$ and growth determined at $595 \mathrm{~nm}$. Plates were washed,

208 fixed, and stained after which biofilm (eluded crystal violet) was determined at $570 \mathrm{~nm}$.

209 Time-course assays were set up independently in triplicate using drug concentrations

210 prepared in serial 2-fold dilutions ranging from $0.3125 \mu \mathrm{M}$ to $20 \mu \mathrm{M}$ at time points $4,8,12$,

211 18, 24 and $48 \mathrm{~h}$ respectively. To determine antibiofilm spectra, 2 reference strains (042 and

212 60A), 25 EAEC strains identified from cases of childhood diarrhoea in an on-going study in

213 Nigeria [18,33] were used and isogenic EAEC 042 mutants (Table 1) were used to infer

214 mechanisms of action involving known surface factors.

\section{Growth kinetic assay}

217 We monitored growth in the presence of varied concentrations of hit compounds at $37^{\circ} \mathrm{C}$ in 218 sterile 96-well polystyrene plates. Concentrations of compounds were prepared in serial 2219 fold dilutions ranging from $0.3125 \mu \mathrm{M}$ to $20 \mu \mathrm{M}$ in DMEM. Thereafter, serially diluted

220 compounds were added, assay was set in triplicate for each hit concentration and optical 221 density was determined at $595 \mathrm{~nm}$ using a microplate spectrophotometer at 0 mins, 30mins, 222 then hourly up until the $8^{\text {th }}$ hour, incubating at $37^{\circ} \mathrm{C}$ between readings.

\section{Molecular docking}

225 In order to obtain atomic level insight into and to validate the mechanism of action of the 226 identified hits, we employed a molecular docking protocol that screened the identified hit 227 molecules against the anti-aggregation protein (Aap) [36], the likely antibiofilm target from 228 our mutant and compliment testing assays. The employed Aap structure (accession code 229 2JVU.pdb [36]), solved using solution NMR, comprises 20 structurally distinct conformers 
230 all of which were individually employed as receptor molecules, a strategy that allowed for the

231 incorporation of macromolecular flexibility in the docking protocol. For the docking

232 screening, three dimensional models were first generated for the five identified MMV

233 molecules using the ChemBioOffice Suite. Energy minimization with the steepest descent

234 algorithm was then performed on each model to resolve steric clashes and identify each

235 molecule's potential energy minimum from the sampled conformational landscape. Each

236 model was then saved in the $\mathrm{P}$ rotein $\mathrm{D}$ ata $\mathrm{B}$ ank (PDB) format.

237 Gasteiger atomic charges, needed for a more accurate computation of electrostatics of the

238 binary interactions, were calculated using AutoDock Tool [37,38] for each of the five MMV

239 hits as well as for each of the twenty Aap structures. AutoDock was also employed in setting

240 up a hyperrectangular docking grid with $\mathrm{x}, \mathrm{y}, \mathrm{z}$ dimension (in Ang strom units) of

$24147.25,36.0,36.0$ centred at $72.234,0.223,1.139$, respectively. The grid dimensions were

242 carefully selected to achieve a total coverage of the Aap models. Using AutoDock Vina [39],

243 each of the five hit compounds was subsequently subjected to docking screening against each

244 of the twenty Aap conformers using a rigid docking protocol with bonded degrees of freedom

245 DOFs) frozen in the receptor macromolecules while all torsional DOFs in the five hit

246 compounds were geometrically optimized during conformational search for stable ligand-

247 receptor complexes. The best binding free energies computed for each of the five hits from

248 this ensemble docking approach were then averaged over the twenty Aap conformations.

249 A similar docking was performed for subunits of the aggregative adherence fimbriae variant

250 II (AAF/II), a previously reported antibiofilm targeted in EAEC 042 by nitazoxanide [28]; the

$251 \mathrm{AAF} / \mathrm{II}$ is suspected as a second target for one of the hits from the outcome of the 042 mutant

252 testing. The solution NMR spectroscopy structure of AAF/II major subunit (accession code

253 2MPV [8]), and the dimerized crystallographic (3.0 $\AA$ ) AAF/II minor subunit (accession code

254 4OR1 [8]) were retrieved from www.rcsb.org. The five hits and NTZ were docked against

255 the macromolecular structure of the AAF/II using docking grid with xyz dimensions (unit of 
$256 \AA$ A) of $12.890,11.530,11.697$ and centred at $13.002,1.147,-9.750$ for the AAF/II major

257 subunit. In the case of the minor subunits xyz dimensions of 8.765, 11.831, 11.142 and

258 centred at $-22.572,51.865,-2.643$ were employed.

\section{Complementing the aap mutant}

260 PCR primers were designed to amplify the aap (antiaggregative protein) gene from 042

261 genomic DNA. The sequence of primers used were: GGCcaattgatgaaaaaaattaagtttgttatcttttc 262 and GATCCCTGCAGGttatttaacccattcggttagag with MfeI (Cat. No. R0589S) and SbfI (Cat.

263 No. R0642S) restriction enzyme recognition sites incorporated into the tails respectively for 264 cloning into pMAL-c5X vector (NEB, Cat. No. N8018S). The aap gene was amplified using 265 the following cycle: $94^{\circ} \mathrm{C}$ for 2 min, then 25 cycles of $94^{\circ} \mathrm{C}$ for $30 \mathrm{sec}, 55^{\circ} \mathrm{C}$ for $45 \mathrm{sec}$ and $26672^{\circ} \mathrm{C}$ for $30 \mathrm{sec}$ followed by a final extension of $72^{\circ} \mathrm{C}$ for 15 mins. PCR products were 267 purified using Zymo DNA Clean \& Concentrator ${ }^{\mathrm{TM}}-5$ Kit (Cat. No. D4003), digested with $268 M f e I$ and SbfI, and ligated into pMAL-c5X vector using T4 ligase (Cat. No. M0202S). The 269 recombinant plasmid obtained, was cloned into ER2523 (NEB express, Cat. No. E4131S) and 270 resistant clones were isolated on LB-ampicillin plates $(100 \mu \mathrm{g} / \mathrm{ml})$. The resulting clone, 271 pDAK24, was verified using PCR to confirm the presence of the aap insert in plasmid, 272 extracted using the QIAprep ${ }^{\circledR}$ Spin Miniprep Kit (Cat. No. 27106), and sequenced. The 273 verified pDAK24 clone was used to transform LV1 (042_aap), LV2 (042 Aaap4hral) and

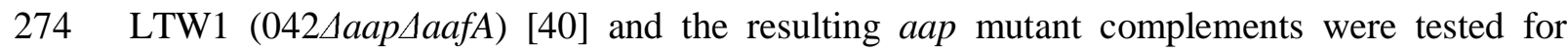
275 biofilm formation and inhibition.

\section{Comparative analysis of virulence genes}

277 Antibiofilm spectra of the five hits identified in this study were investigated using EAEC 278 reference strain 042 [33], 60A from Mexico [41] and 25 other EAEC strains identified and 279 characterized in our laboratory in Nigeria from an ongoing diarrhoea case-control study [18].

280 Virulence gene profiles of strains in each group were retrieved from whole genome sequence 
281 data using VirulenceFinder database [42] then compared to identify genes which were unique

282 to each group for each hit.

\section{Statistical analysis}

285 Data from biofilm inhibition assays (average of three replicates) were analyzed by comparing 286 inhibition / percentage inhibitions for tests and controls and significant differences were 287 inferred from Fisher's exact test (to identify genes which are unique to strains whose biofilms 288 were inhibited by hits) and student's t-test analysis (for biofilm inhibition assay).

\section{Results}

\section{Pathogen Box contains compounds capable of inhibiting EAEC biofilm formation}

293 The 400-compound Pathogen Box chemical library was screened for EAEC biofilm

294 formation inhibitors in a medium throughput assay. Initial hit selection criteria were that

295 molecules must demonstrate at least $30 \%$ biofilm inhibition and under $10 \%$ growth inhibition

296 at a concentration of $5 \mu \mathrm{M}[43,44]$. Applying these criteria, we identified five compounds

297 which reproducibly inhibited accumulation of biofilm biomass by EAEC 042 and/ or

298 MND005E by 30-85\% while inhibiting growth by $\leq 10 \%$ (Figure 1a-d). As shown in Table

299 1, the compounds possessed diverse molecular architectures and chemical properties.

300 Consistent with the known heterogeneity of EAEC strains, the two EAEC test strains, which

301 were selected because they were phylogenetically distant and had largely non-overlapping

302 suites of virulence factors, retrieved different hits from the library. Compounds

303 MMV687800, MMV688978 and MMV687696 inhibited biofilm formation of strain 042

304 only, MMV000023, was active against MND005E while MMV688990 showed activity

305 against both strains. Nitazoxanide (MMV688991), the only previously reported EACE 042

306 biofilm inhibitor [28], is also contained within Pathogen Box but did not meet our hit criteria. 
307 Significant antibiofilm activity was previously reported for NTZ at $15 \mu \mathrm{g} / \mathrm{ml}(48.8 \mu \mathrm{M}), 20$

$308 \mu \mathrm{g} / \mathrm{ml}(65.15 \mu \mathrm{M})$ and $25 \mu \mathrm{g} / \mathrm{ml}(81.45 \mu \mathrm{M})$ with an estimated growth inhibition of up $50 \%$

309 [28]. Based on this information, we conducted comparative biofilm inhibition assays for our

310 hits (at $5 \mu \mathrm{M}$ ) and $\mathrm{NTZ}$ at $48.8 \mu \mathrm{M}, 65.15 \mu \mathrm{M}$ and $81.45 \mu \mathrm{M}$. We observed similar patterns

311 of antibiofilm activity and growth inhibition with NTZ at these concentrations as reported

312 earlier by Shamir et al. [28]. Additionally, the five validated hits in this screen were found

313 active at concentrations at least 10 times lower than those of nitazoxanide (NTZ) as shown in

314 Figure 1c. Consequently, hits from this screen are potent biofilm inhibitors and we cannot

315 rule out the presence of other weak biofilm inhibitors in Pathogen Box (Figure 1a-b). The

316 class (medicinal indication) of the 400 Pathogen Box compounds matched with biofilm

317 inhibition outcome is summarized in Table S1. Four of the five validated hits are reference

318 compounds with other known activities, while one is indicated for tuberculosis. The three

319 unvalidated hits (which were not retrieved consistently in confirmatory assays especially in

320 concentration dependent and growth kinetics assays applying our hit selection criteria) in this

321 study are kinetoplastid inhibitors.

323 In the course of the screen, we observed that known antibacterials in Pathogen Box, 324 doxycycline, levofloxacin and rifampicin inhibited biofilm formation by $60.33,89.92$, and $32590.08 \%$ respectively but also inhibited growth by $15.07,64.53$ and 32.53 . Our initial 326 preliminary screens additionally turned out MMV688362, MMV688771 and MMV676159 as

327 EAEC 042 hits. However repeated testing and further validation in confirmatory assays 328 revealed antibiofilm activity were due to antibacterial activity in one case, with the others 329 exhibiting a little lower antibiofilm activity than the hit criteria minimum cut off (30\%). Five 330 additional compounds also inhibited both growth and biofilm formation and are being further 331 evaluated as potential antibacterial hits. 
333 Planktonic cell growth of EAEC 042 and MND005E in the presence of $0.3125-20 \mu \mathrm{M}$ of

334 hits over a time course of 0 to $8 \mathrm{~h}$ was not significantly different from the compound-free 335 control (Figure 2).

337 We tested concentration-dependency of three of our five hits, MMV687800, MMV688978 338 and MMV688990 against EAEC strain 042. As shown in Figure 3, the three compounds 339 exhibited concentration-dependent inhibitory effects on biofilm formation in EAEC 042 with 340 correlations $\left(\mathrm{r}^{2}\right)$ of $0.9552,0.9355$ and 0.9593 for hit MMV688978, MMV687800,

341 MMV688990 respectively. Individual analysis of concentration-dependent curves revealed 342 that hits exhibited potent activity, with concentrations as low as $2.5 \mu \mathrm{M}$ inhibiting biofilm 343 formation by up to $30 \%$ (Figure 3).

344 When we tested inhibition of biofilm formation across a $48 \mathrm{~h}$ time-course, we found that the 345 biofilm inhibition effect was most pronounced at early time points and then again after the 346 biofilm was well established (Figure 4) and (Figure S1).

348 Inhibition of biofilm formation in 042 aap, hral and aaf mutants

349 As a first step towards identifying mechanisms of action, we tested biofilm inhibition in 350 single and double mutants in three key colonization genes of EAEC strain 042 against the 351 four hits that inhibit biofilm formation in this strain. MMV687696, MMV688978 and 352 MMV688990 significantly inhibited biofilm formation by all five mutants at a level similar to 353 wildtype 042 inhibition (Figure 5b-d). Noteworthily, MMV687800 reduced biofilm 354 formation by $042 \Delta h r a l$ (SB1) to a similar degree as the wildtype (EAEC 042) (Figure 5a), 355 ruling out Hra1 as a target for this compound. A slightly lower degree of inhibition was seen 356 in the $042 \Delta a a f A$ mutant (3.4.14) but this was not statistically significantly different from the 357 inhibition produced on the wild type (Figure 5a). For the aap mutant, 042 $\triangle a a p$ (LV1), 358 inhibition occurred to a significantly lower degree. When we tested aap double mutants, we 


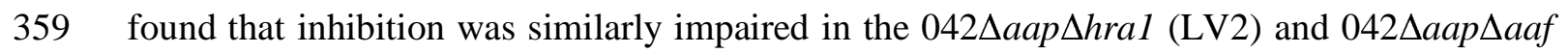

360 (LTW1), with the latter showing no significant difference $(\mathrm{p}>0.05)$ in biofilm formation in

361 the presence or absence of MMV687800 (Figure 5a). Thus, the data point to aap as a likely

362 target for MMV687800 and aaf may or may not be a minor target. Following the hypothesis

363 that the antibiofilm activity of MMV687800 could involve aap, we set up biofilm assays with

364 MMV687800 against EAEC 042, LV1, LV2, LTW1 alone and with the mutants

365 complemented with pDAK24. As is known for these strains, biofilm inhibition was low in

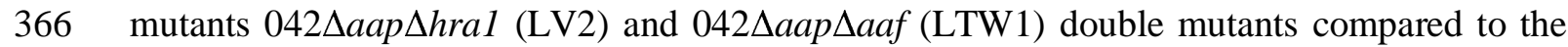

367 wild type strain 042 (Figure 6). Conversely, notable reductions in biofilm formation were

368 observed in the construct, LV1(pDAK24), LV2(pDAK24) and LTW1(pDAK24) (Figure 6),

369 where aap deletions were complemented in trans.

370 Inhibition was not completely abrogated in aap mutants, hence it is probable that there is

371 more than one MMV687800 target in 042 and collectively, the data suggest that aafA could

372 represent a second target.

373

374 Antibiofilm spectra of hit compounds

375 Along with the two EAEC strains used in our preliminary screen (EAEC strains 042 [33] and

376 MND005E [18]), we investigated antibiofilm activity of hits against, 60A an EAEC isolate

377 from Mexico [39.41] and 24 EAECs strains from our laboratory [18], which form moderate

378 or strong biofilms and have been whole genome sequenced (Table 2). All five hits inhibited

379 biofilms in different EAEC strains to varying degrees with MMV687800, MMV688978 and

380 MMV688990 demonstrating broader antibiofilm activity spectra and likely different

381 mechanisms of action (Table 3). Biofilm formation was inhibited by MMV687800 for all five

382 isolates carrying allele 3 of the aap gene present in 042 , but only four of 22 strains carrying

383 other aap alleles or no aap gene ( $\mathrm{p}=0.0016$, Fisher's exact test). Similarly, MMV687800 
384 inhibited biofilms in all five strains bearing aafA, aafB, aafC and aafD genes but only four of

38522 strains carrying none of the aaf genes $(\mathrm{p}=0.0016$, Fisher's exact test).

\section{Binding affinity of hit compounds with Aap and Aaf subunits}

388 To validate Aap as a target for antibiofilm activity by MMV687800 and to determine whether

389 AAF/II might be a target, we independently computed binding affinities of all five hits and

390 NTZ (first reported to interfere with AAF/II and type I pili assembled by the chaperone usher

391 pathway in EAEC) [28] with twenty Aap conformers and the AAF/II major and minor

392 subunits using docking simulation. MMV687696 and MMV687800 demonstrated

393 exceptionally strong interaction (in some cases $\Delta \mathrm{G} \leq-8.1 \mathrm{kcal} / \mathrm{mol}$ ) with Aap as depicted in

394 Figure 7. The other 3 hits exhibited comparatively lower affinity for Aap. As expected, (NTZ

395 does not target Aap), there was also significantly lower affinity for Aap with NTZ (Figure 7).

397 Since data from the isogenic 042 mutant biofilm inhibition assay additionally suggests that MMV687800 could have a second target (likely AAF/II), all five investigated hits were docked against AAF/ II major and minor subunits. The five hits and NTZ demonstrated different degrees of moderately thermodynamically favourable binding to the EAEC (AAF/II major and minor subunits), the only EAEC adhesins whose solution structure have been resolved [8]. Hits demonstrated stronger binding to non-Gd site cavities present on the

403 surfaces of the EAEC proteins (Table 4). NTZ, initially shown to inhibit aggregative 404 adherence fimbria and type I pili assembled by the Chaperone Usher (CU) pathway in EAEC 405 [28] was subsequently proven to interfere with the folding of the usher beta-barrel domain in 406 the outer membrane [45]. It was recently reported for its selective activity in disrupting beta407 barrel assembly machine (BAM)-mediated folding of the outer membrane usher protein in 408 uropathogenic Escherichia. coli (UPEC) [46]). In our screening it demonstrated the highest 409 affinity $(-6.6 \mathrm{kcal} / \mathrm{mol})$ for AAF/II closely followed by MMV687800 with $\Delta \mathrm{G}$ of -5.7 
$410 \mathrm{kcal} / \mathrm{mol}$. With focused binding interaction (that is, focusing on the Gd site), the strongest

411 binding interaction with AAF/II major subunit among the hits was obtained with

412 MMV687800 (-5.7 kcal/ mol), MMV000023 (-5.5 kcal/mol), then MMV688978 (-5.2

$413 \mathrm{kcal} / \mathrm{mol}$ ) (Table 4). In all, Nitazoxanide, reported to interfere with AAF/ II assembly [28],

414 outperformed all five test compounds with respect to the strength of interaction with the AAF

415 /II binding site even though the differences are at best marginal.

416

417 


\section{Discussion}

419 Biofilms contribute significantly to pathogenesis of several bacterial infections [47-51]. They

420 are major players thwarting host defense and the activity of antimicrobials against many

421 microorganisms [52] and will consequently require a novel approach to target them during

422 infections. EAEC are known to form copious biofilms, which enhances their persistence

423 during infection [53,54] hence antibiofilm agents could be particularly effective [55]. As

424 antibiofilm agents attack a colonization process and not bacterial viability, it is hoped that,

425 unlike conventional antibiotics, they will exert less selection pressure for antimicrobial 426 resistance [56].

428 Whilst antibiofilm activity of a few synthetic and natural small molecules has been 429 demonstrated in vitro [52,57-62] and in vivo [55,63,64], for other pathogens, only one study 430 reported significant antibiofilm activity of a compound, nitazoxanide, against EAEC biofilms 431 [28]. Shamir et al (2010) discovered the antibiofilm activity of NTZ when they were 432 evaluating its growth inhibitory activity and subsequently showed that this antiparasitic 433 compound inhibits assembly of AAF/II. NTZ has subsequently been shown to interfere with 434 pilus usher function [45,46] and Bolick et al (2013) [55] were able to show that it reduces 435 diarrhoea in vivo, and shedding of EAEC at concentrations not inhibiting growth. These data 436 suggest that antiadhesive agents have therapeutic potential against EAEC. By systematically 437 screening for EAEC antibiofilm activity, this study has uncovered many more inhibitors that 438 are significantly more potent than NTZ. At least one of them, MMV687800 inhibits EAEC439 specific targets and so is unlikely to have deleterious effects on the normal flora.

441 The five hits we identified from the MMV's pathogen box reproducibly inhibited biofilm 442 formation by one or two EAEC strains by $30-85 \%$ while inhibiting growth by $\leq 10$ at $5 \mu \mathrm{M}$. 443 Our hit rate for biofilm inhibitors that do not inhibit growth (1.25\%), from a curated library, 
444 provides support for this drug discovery approach. Targeting EAEC biofilms without

445 compromising their viability holds a high potential since hits from our screen are unlikely to 446 select for antibacterial resistance due to selection pressure for resistant strains.

447 We report here antibiofilm activity of five compounds, most of which are not new to the drug 448 industry thus providing a good starting point for antibiofilm drug discovery. Four of our hits 449 MMV687800, MMV688978, MMV000023, and MMV688990 are existing therapies for 450 other neglected tropical diseases, but not diarrhoea or Gram-negative infections. Clofazimine 451 (MMV687800) is used to treat leprosy (Mycobacterium leprae): 452 https://www.drugbank.ca/drugs/DB00845 and its antimicrobial spectrum does not include 453 Gram negative organisms like EAEC $[65,66]$. The anti-tubercular drug was recently reported 454 to demonstrate a broad activity spectrum against coronaviruses including SARS-CoV-2 [67]. 455 Miltefosine (MMV688990) is an anti-leishmanial drug [68] originally developed as an anti456 cancer drug in 1980s: https://www.drugbank.ca/drugs/DB09031, Auranofin (MMV688978) is 457 a gold salt used in treating rheumatoid arthritis: https://www.drugbank.ca/drugs/DB00995 but 458 is now been tried for several other disease conditions, not including diarrhoea [69]. 459 Primaquine (MMV000023) is an agent: 460 https://www.drugbank.ca/drugs/DB01087, which is active against gametocytes as well as 461 recalcitrant parasitic forms of malaria, and therefore useful for preventing vivax and ovale 462 relapse [70]. None of these four compounds has any previously documented antibacterial 463 activity (in vivo or in vitro) against Gram negative bacteria, however, Ugboko et al (2019) in 464 an in silico screen and analysis of broad-spectrum molecular targets and lead compounds for 465 potential anti-diarrhoea agents reported MMV687800 as one of the compounds with 466 predicted activity against potential targets for diarrhoea disease among twenty five other 467 compounds [71]. Low et al (2017) in screen to identify TB active compounds against 468 nontuberculous Mycobacterium additionally retrieved MMV687800 as an M. avium-specific 
469 main hits among five other MMV compounds [72] and Hennessey et al (2018) also reported

470 MMV687800 among seven MMV compounds as an inhibitor with dual efficacy against

471 Giardia lamblia and Cryptosporidium parvum in a pathogen box screen [73].

472 Rollin-Pinheiro et al (2021) in a screen to identify antifungal drugs against Scedosporium and

473 Lomentospora species from the pathogen box chemical library retrieved MMV688978 as hit

474 in addition to discovering its ability to decreasee the fungal biomass of preformed biofilm by

475 about $50 \%$ of at $1 \times$ MIC for $S$. aurantiacum and $70 \%$ at $4 \times$ MIC of $S$. dehoogii and $L$.

476 prolificans [74]. MMV687696, has been reported by MMV to possess anti-tuberculosis 477 activity.

478 The antiparasitic compound nitazoxanide, also a component of Pathogen Box, which was 479 previously reported to inhibit biofilm by EAEC 042 [28] was not recovered as hit from our 480 screen and we verified that it is indeed inactive at $5 \mu \mathrm{M}$, our screen concentration. We re481 tested nitazoxanide at concentrations for which antibiofilm activity was previously recorded 482 and observed significant concentration-dependent biofilm inhibition at 15, 20 and $25 \mu \mathrm{g} / \mathrm{ml}$ $483(48.8,65.15$ and $81.4 \mu \mathrm{M})$ but with estimated growth inhibition of up to 50\% [28]. 484 Consequently, the validated hits obtained from the screen in this study, which lack growth 485 inhibition activity at the concentration tested, were at least 10 times more active in biofilm 486 inhibition than nitazoxanide the only known EAEC biofilm inhibitor, which has subsequently 487 been shown to be effective against experimental infections in a weaned mouse model [55]. 488 Unlike NTZ, our five hits inhibited biofilm formation at concentrations that did not produce 489 significant growth inhibition, pointing to the possibility of biofilm-specific targets and 490 minimal, if any, cross resistance with clinical antibacterials [44,56]. For three of the 491 compounds for which we could get sufficient chemical to test, inhibition was largely 492 concentration dependent, again suggesting that specific biofilm factors are targeted. When 
493 those factors are EAEC-specific, use of antibiofilm agents is unlikely to disrupt the normal

494 flora, including other E. coli, which are protective against enteric infection.

495 Biofilm formation is a complex and stepwise process involving numerous bacterial factors, 496 which vary among and even within pathotypes. Early-stage contributors include adhesins,

497 flagella and secreted protein autotransporters. At late-exponential phase, the accumulation of 498 quorum sensing signals leads to the activation of other genes. Late-stage biofilm factors 499 include adhesins with greater permanence, components that comprise or requite a 500 macromolecular matrix as well as antiagregation proteins which can be co-opted to release 501 bacteria from the biofilm.

503 The temporal patterns of biofilm inhibition and EAEC inhibition spectra of compounds 504 MMV687800, MMV688978, MMV687696, MMV000023 and MMV688990 were different, 505 implying that they likely target different contributors to EAEC biofilm formation. EAEC 506 expressed many surface factors involved in host adherence and biofilm formation $507[21,40,75.76]$. Any of these, or their regulators, could be direct targets. Overlaying activity 508 spectra data with virulence factor profiles of genome sequenced EAEC strains provided 509 preliminary insights to mechanism of action. For MMV687800, subsequent testing of five 510 isogenic mutants of EAEC 042 provided further insight. Aap is an antiaggregation protein or 511 dispersin that allows bacteria to detach from old biofilms and seed new ones. Mutants in aap 512 show increased biofilm formation but impaired colonization [21,40]. Biofilm formation by 513 aap mutant (LV1: 042 Aaap) was significantly less inhibited by MMV687800 than wildtype.

514 Additionally (LV2: 042 Аaap_hral) double mutants [40] were inhibited in biofilm formation 515 to a proportionally lower degree and inhibition seen in (LTW1: 042 Aaap_aaf) was 516 statistically insignificant compared to controls no compound at all $(\mathrm{p}=0.11)$. This 517 phenotype could be complemented in trans and thus molecular Koch's postulates [77] are 518 fulfilled for aap as an MMV687800 target. 
520 To preliminarily determine whether the interaction between MMV687800 and Aap could be 521 direct, we independently determined binding affinities of hits with twenty Aap conformers 522 using molecular docking techniques since the solution structure of Aap has been resolved by 523 NMR [35]. The strong binding interaction sustained for hit MMV687800 and in the outcome 524 of the molecular computational docking experiments against 20 conformational instances of 525 the dispersin (Aap) in Figure 7 indicates a high significance for the obtained affinities.

526 MMV687800 demonstrated moderately strong interaction (in some cases $\Delta \mathrm{G} \leq-8.1 \mathrm{kcal}$ )

527 with Aap. This additionally suggests that aap is one of the surface factors targeted by 528 MMV687800.

529 The aafA gene encodes the structural subunit of AAF/II fimbriae and Hra1, the heat-resistant 530 agglutinin 1 is an outer-membrane protein involved in autoaggregation that serves as an 531 accessory colonization factor [40,75,78]. MMV687800 showed a small, insignificant 532 reduction of activity in the aafA mutant 3.4.14 [79], but not the hral mutant, which we 533 initially discounted, given the robust phenotype with Aap. However, the reduction in 534 MMV687800 biofilm inhibition was visible with the aap aafA mutant LTW1, and 535 complementation of this mutant with aap alone LTW1(pDAK24) could not fully restore 536 biofilm inhibition. These data, and the comparative genomic data which shows that aafA-D 537 are unique to biofilm inhibition groups alone (data not shown), strengthen the likelihood that 538 AAF/II is implicated in MMV687800 biofilm inhibition, albeit to a lower degree than Aap.

539 The five hit compounds demonstrated stronger binding to non-Gd site cavities present on the 540 surfaces of AAF/II (Table 4). Docking outcome however revealed NTZ, known to inhibit 541 AAF/II assembly exhibited highest affinity $(-6.6 \mathrm{kcal} / \mathrm{mol})$ for AAF/II followed by 542 MMV687800 which had $-5.7 \mathrm{kcal} / \mathrm{mol}$ again, indicating that AAF/II could be a likely target 543 for MMV687800. 
544 This study has some limitations. EAEC are highly heterogeneous and the full spectrum of

545 lineages and virulence factors is not covered by the two strains used for this screen, or even

546 by the 27 strains employed to better understand activity profiles of the hits. We determined

547 the probable mechanism of action of only one hit, taking advantage of the easily-generated

548 VirulenceFinder output and the bank of mutants we had on hand. In doing so, we were able

549 to generate proof of principle and rule out Aap, AAF/II and Hral as targets for the other

550 compounds. However, it is conceivable that at least some of the targets of the other

551 compounds may not be unique to EAEC or may be genes of unknown function. More

552 intensive and unbiased comparative genomic approaches, currently underway, will be needed

553 to exhaustively screen for the targets of the other four hits, which could well be more

554 promising than the hit highlighted in this study.

556 In conclusion, this study identified five biofilm inhibiting but non-growth inhibiting

557 compounds that have not been previously described as bacterial anti-adhesins or Gram-

558 negative antibacterials. Hits discovered from this screen will add to the drug discovery

559 pipeline for this neglected pathogen, improve understanding on EAEC colonization and

560 enhance EAEC-based interventions. Additionally, the understandings from our experiments

561 that some of our hits are unlikely to target known EAEC adhesins is initiating investigations

562 at the molecular level which can open us up to a world of novel adhesins and consequently

563 enhance our understanding of EAEC pathogenic factors. 


\section{Acknowledgement}

574 The authors are grateful to the following people for technical assistance during the project:

575 Jeremiah J. Oloche, Olabisi C. Akinlabi, Jesuferanmi Igbinigie, Erkison Ewazimo Odih,

576 Anderson O Oaikhena, Taiwo Badejo, Adewole D Pelumi, Stella Ekpo, Abiodun Oyerinde,

577 Catherine Oladipo, Emmanuel Bamidele, El-Shama Q Nwoko, Amos Olowokere, Uchechi

578 Okoroafor and Joy Olorundare.

579 We thank the Medicines for Malaria Venture (MMV) for supplying Pathogen Box,

580 supplementary amounts of select chemicals from the box, as well as for drug discovery

581 training for DAK. We additionally thank the Wellcome Trust for advanced training in drug 582 discovery for DAK.

$585 \quad$ Funding

586 This work was supported by an African Research Leader award from the UK Medical 587 Research Council (MRC) and the UK Department for International Development (DFID) 588 under the MRC/DFID Concordat agreement that is also part of the EDCTP2 program 589 supported by the European Union as well as by the National Science Foundation award \#

5901329248 'RUI: Aggregation and Colonization Mediated by Bacterial Colonization Factors' 591 both to INO.

592 Additional support for this project was gratefully received from Grand Challenges Africa 593 (Award \#GCA/DD/rnd3/021), a programme of the African Academy of Sciences 594 (AAS) implemented through the Alliance for Accelerating Excellence in Science in Africa 595 (AESA) platform, an initiative of the AAS and the African Union Development Agency 
596 (AUDA-NEPAD). For this work, GC Africa is supported by the African Academy of

597 Sciences (AAS), Bill \& Melinda Gates Foundation (BMGF), Medicines for Malaria Venture

598 (MMV), and Drug Discovery and Development centre of University of Cape Town (H3D).

599

600 Competing interests

601 The authors have no competing interests to declare.

602

603

604

605

606

607 Author Contributions

608

609 Conceptualization: Iruka N. Okeke.

610 Data curation: David A. Kwasi, Chinedum P. Babalola, Olujide O. Olubiyi and Iruka N. Okeke.

612 Formal analysis: David A. Kwasi, Olujide O. Olubiyi and Iruka N. Okeke.

613 Investigation: David A. Kwasi, Jennifer Hoffmann, Olujide O. Olubiyi and Iruka N. $614 \quad$ Okeke.

615 Methodology: David A. Kwasi, Jennifer Hoffmann, Olujide O. Olubiyi, Ikemefuna C. $616 \quad$ Uzochukwu and Iruka N. Okeke.

617 Resources: $\quad$ Iruka N Okeke, Olujide O. Olubiyi

618 Supervision: Olujide O. Olubiyi, Chinedum P. Babalola and Iruka N. Okeke 
619 Validation:

621 Writing-Drafts: David A. Kwasi, Chinedum P. Babalola, Olujide O. Olubiyi,
David A. Kwasi, Chinedum P. Babalola, Ikemefuna C. Uzochukwu, Olujide O. Olubiyi and Iruka N. Okeke.

\section{References}

1. Walker CL, Aryee MJ, Boschi-Pinto C, Black RE. Estimating diarrhea mortality among young children in low and middle income countries. PLoS One 2012; 7(1):e29151. https://doi.org/10.1371/journal.pone. 0029151 PMID: 22235266.

2. Liu L, Johnson HL, Cousens S, Perin J, Scott S, Lawn JE et al. Global, regional, and national causes of child mortality: an updated systematic analysis for 2010 with time trends since 2000. Lancet. 2012; 379 (9832):2151-61. https://doi.org/10.1016/S01406736(12)60560-1 PMID: 22579125.

3. UNICEF 2019. Diarrheoa data: Diarrheoa as cause of death in children under 5. https://data.unicef.org/resources/dataset/diarrhoea/. Accessed $5^{\text {th }}$ October, 2019.

4. Institute for Health Metrics and Evaluation (IHME) Global burden of disease, 2018. Annual deaths from diarrheal diseases differentiated by age categories. Retrieved from https://ourworldindata.org/diarrheal-diseases on 29th January, 2020.

5. Acosta AM, De Burga RR, Chavez CB, Flores JT, Olortegui MP, Pinedo SR et al. Relationship between growth and illness, enteropathogens and dietary intakes in the first 2 years of life: findings from the MAL-ED birth cohort study. BMJ Glob Health 2017; 2(4):e000370. https://doi.org/10.1136/bmjgh-2017-000370 PMID: 29333282.

6. Roche JK, Cabel A, Sevilleja J, Nataro J, Guerrant RL. Enteroaggregative Escherichia coli (EAEC) impairs growth while malnutrition worsens EAEC infection: a novel murine model of the infection malnutrition cycle. J Infect Dis. 2010;202:506514. DOI: $10.1086 / 654895$.

7. Kotloff KL, Nataro JP, Blackwelder WC, Nasrin D, Farag TH, Panchalingam S et al. Burden and aetiology of diarrhoeal disease in infants and young children in developing countries (the Global Enteric Multicenter Study, GEMS): a prospective, case-control study. Lancet 2013; 382(9888):209-22. https://doi.org/10.1016/S01406736(13)60844-2 PMID: 23680352.

8. Berry AA, Yang Y, Pakharukova N, Garnett JA, Lee W, Cota E, Marchant J, Roy S, Tuittila M, Liu B, Inman KG, Ruiz-Perez F, Mandomando I, Nataro JP, Zavialov AV, Matthews S. Insight into Host Recognition by Aggregative Adherence Fimbriae of Enteroaggregative Escherichia coli. Plos Pathogen 2014; 10(9): e1004404. https://doi.org/10.1371/journal.ppat.1004404.

9. Harrington SM, Dudley EG, Nataro JP. Pathogenesis of enteroaggregative Escherichia coli infection. FEMS Microbiol Lett. 2006; 254:12-8; PMID:16451

10. Scavia G, Staffolani M, Fisichella S, Striano G, Colletta S, Ferri G, Escher M, Minelli F, Caprioli A. Enteroaggregative Escherichia coli associated with a foodborne outbreak of gastroenteritis. J Med Microbiol. 2008; 57: 1141-1146. https://doi.org/10.1099/jmm.0.2008/001362-0 PMID: 18719185.

11. Acosta GJ, Vigo NI, Durand D, Riveros M, Arango S, Zambruni M, and Ochoa TJ. Diarrheagenic Escherichia coli: Prevelence and Pathotype Distribution in Children from Peruvian Rural Communities. Am. J. Trop. Med Hyg. 2016; 95 (3); 574-579. 
12. Cohen MB, Hawkins JA, Weckbach LS, Staneck JL, Levine MM, Heck JE. Colonization by Enteroaggregative Escherichia coli in travelers with and without diarrhea. J Clin Microbiol. 1993; 31: 351-353. PMID: 8432822.

13. Steiner TS, Lima AA, Nataro JP, Guerrant RL. Enteroaggregative Escherichia coli produce intestinal inflammation and growth impairment and cause interleukin-8 release from intestinal epithelial cells. J Infect Dis 1998; 177(1):88-96. PMID: 9419174.

14. Rogawski ET, Platts-Mills JA, Seidman JC, John C, Mahfuz M, Ulak M, Shrestha SK, Soofi SB, Yori PP, Mduma E, Svensen E, Ahmed T, Lima AAM, Bhutta ZA, Kosek MN, Lang DR, Gottlieb M, Zaidi AKM, Kang G, Bessong PO, Houpta ER, Guerranta RL. Use of antibiotics in children younger than two years in eight countries: a prospective cohort study. Bull World Health Organ 2017;95:49-61 | doi: http://dx.doi.org/10.2471/BLT.16.176123

15. Okeke IN, Lamikanra A, Czeczulin J, Dubovsky F, Kaper JB, Nataro JP. Heterogeneous Virulence of Enteroaggregative Escherichia coli Strains Isolated from Children in Southwest Nigeria. The Journal of Infectious Diseases 2000; 181 : 25260.

16. Okeke IN, Nataro JP. Enteroaggregative Escherichia coli. Lancet Infect Dis. 2001;1(5):304-13.

17. Opintan JA, Newman MJ, Ayeh-Kumi PF, Affrim R, Gepi-Attee R, Sevilleja JE, Roche JK, Nataro JP, Warren CA, Guerrant RL. Pediatric diarrhea in southern Ghana: etiology and association with intestinal inflammation and malnutrition. Am J Trop Med Hyg. 2010;83(4):936-43. doi: 10.4269/ajtmh.2010.09-0792.

18. Akinlabi OC, Ekpo S, Nwoko EQ, Ladipo CO, Adepoju A, Dougan G, Okeke IN. Identification of Enterotoxigenic Escherichia coli from infants attending healthcare centers in Ibadan north. American Society of tropical Medicine and Hygiene Book of Abstracts, 2019; $101: 139-140$.

19. Moran M, Guzman J, Ropars A, McDonald A, Jameson N, Omune B, Ryan S, Wu L. "Neglected disease research and development: how much are we really spending?" PLoS Med. 2009; 6(2): e30.

20. Okeke IN. "Diarrheagenic Escherichia coli in sub-Saharan Africa: status, uncertainties and necessities." J Infect Dev Ctries. 2009; 3(11): 817-842.

21. Sheikh J, Czeczulin JR, Harrington S, Hicks S, Henderson IR, Le Bouguenec C, Gounon P, Phillips A, Nataro JP. A novel dispersin protein in enteroaggregative Escherichia coli. J Clin Invest. 2002; 110:1329 -1337. https:// doi.org/10.1172/JCI16172.

22. Huang DB, Okhuysen PC, Jiang ZD, DuPont HL. Enteroaggregative Escherichia coli: an emerging enteric pathogen. Am J Gastroenterol. 2004; 99:383-389.

23. Hicks S, Candy DC, Phillips AD. Adhesion of enteroaggregative Escherichia coli to pediatric intestinal mucosa in vitro. Infect. Immun. 1996; 64:4751-4760.

24. Bangar R and Mamatha B. Identification of enteroaggregative Escherichia coli in infants with acute diarrhea based on biofilm production in Manipal, South India. Indian J. Med. Sci. 2008; 62:8-12. 10.4103/0019-5359.38916.

25. Pereira, AL, Silva TN, Gomes ACMM, Araújo ACG, Giugliano, LGG. Diarrheaassociated biofilm formed by enteroaggregative Escherichia coli and aggregative Citrobacter freundii: a consortium mediated by putative F pili. Microbiology 2010; 10:57.

26. Nataro JP, Deng Y, Cookson S, Cravioto A, Savarino SJ, Guers LD, Levine MM, Tacket CO. Heterogeneity of enteroaggregative Escherichia coli virulence 
demonstrated in volunteers. J Infect Dis. 1995 Feb;171(2):465-8. doi: 10.1093/infdis/171.2.465. PMID: 7844392.

27. Jensen BH, Olsen KEP, Struve C, Krogfelt KA, Petersen AM. Epidemiology and Clinical Manifestations of Enteroaggregative Escherichia coli Clinical Microbiology Reviews 2004; 27(3) : 614 - 630, doi:10.1128/CMR.00112-13.

28. Shamir, ER, Warthan M, Brown SP, Natro JP, Guerrant RL, Hoffman PS. Nitazoxanide inhibits biofilm production and hemagglutination by enteroaggregative Escherichia coli strains by blocking assembly of AafA fimbriae. Antimicrob Agents Chemother 2010; 54: 1526-33.

29. Estrada-Garcia T, Navarro-Garcia F. Enteroaggregative Escherichia coli pathotype: a genetically heterogeneous emerging foodborne enteropathogen. FEMS Immunol Med Microbiol. 2012; 66: 281-298.

30. O'Toole G, Kaplan HB, Kolter R. Biofilm formation as microbial development. Annu Rev Microbiol. 2000;54:49-79. doi: 10.1146/annurev.micro.54.1.49. PMID: 11018124.

31. O'Toole GA. Microtiter dish biofilm formation assay. J Vis Exp. 2011 Jan 30;(47):2437. doi: 10.3791/2437. PMID: 21307833; PMCID: PMC3182663.

32. Wakimoto N, Nishi J, Sheikh J, Nataro JP, Sarantuya J, Iwashita M, Manago K, Tokuda K, Yoshinaga M, Kawano Y. Quantitative biofilm assay using a microtiter plate to screen for enteroaggregative Escherichia coli. Am J Trop Med Hyg. 2004 Nov;71(5):687-90. PMID: 15569806.

33. Nataro JP, Kaper JB, Robins-Browne R, Prado V, Vial P, Levine MM. Patterns of adherence of diarrheagenic Escherichia coli to HEp-2 cells. Pediatr Infect Dis J. 1987 Sep;6(9):829-31. doi: 10.1097/00006454-198709000-00008. PMID: 3313248.

34. Chaudhuri RR, Sebaihia M, Hobman JL, Webber MA, Leyton DL, et al. Complete Genome Sequence and Comparative Metabolic Profiling of the Prototypical Enteroaggregative Escherichia coli Strain 042. PLoS ONE 2010; 5(1): e8801. doi:10.1371/journal.pone.0008801.

35. Amin, A. G., Angala, S. K., Chatterjee, D., \& Crick, D. C. (2009). Rapid screening of inhibitors of Mycobacterium tuberculosis growth using tetrazolium salts. Methods in molecular biology (Clifton, N.J.), 465, 187-201.

36. Jorge J. Velarde, Kristen M. Varney, Keith G. Inman, Mauricio Farfan, Edward Dudley, Jonathan Fletcher, David J. Weber and James P. Nataro. Solution structure of the novel dispersin protein of enteroaggregative Escherichia coli. Molecular Microbiology 2007; 66(5), 1123-1135 doi:10.1111/j.1365-2958.2007.05985.x..

37. Goodsell D, Morris G, Olson A. Automated docking of flexible ligands: Applications of AutoDock. J. Mol. Recognit. 1996; 9: 1-5.

38. Santos-Martins, D, Forli S, Ramos M, Olson A. AutoDock4 Zn: An Improved AutoDock Force Field for Small-Molecule Docking to Zinc Metalloproteins. J. Chem. Inf. Model. 2014; 54: 2371-2379.

39. Trott O, \& Olson A.J. AutoDock Vina: improving the speed and accuracy of docking with a new scoring function, efficient optimization, and multithreading. Journal of computational chemistry, 2010; 31(2), 455-461.

40. Blanton LV, Wang LT, Hofmann J, DuBow J, Lafranc A. Aggregative Adherence and Intestinal Colonization by Enteroaggregative Escherichia coli Are Produced by Interactions among Multiple Surface Factors https://doi.org/10.1128/mSphere.0007818. American Society for Microbiology Msphere 2018; 3(2) e00078-18.

41. Czeczulin JR, Whittam TS, Henderson IR, Navarro-Garcia F, Nataro JP. Phylogenetic analysis of enteroaggregative and diffusely adherent Escherichia coli. Infect Immun. 
1999 Jun;67(6):2692-9. doi: 10.1128/IAI.67.6.2692-2699.1999. PMID: 10338471; PMCID: PMC96572.

42. Joensen KG, Scheutz F, Lund O, Hasman H, Kaas RS, Nielsen EM, Aarestrup FM. Real-time whole-genome sequencing for routine typing, surveillance, and outbreak detection of verotoxigenic Escherichia coli. J Clin Microbiol. 2014 May;52(5):150110. doi: 10.1128/JCM.03617-13. Epub 2014 Feb 26. PMID: 24574290; PMCID: PMC3993690.

43. Rebollo-Lopez MJ, Lelièvre J, AlvarezGomez D, Castro-Pichel J, Martínez-Jiménez F, Papadatos G, et al. Release of 50 new, drug-like compounds and their computational target predictions for open source anti-tubercular drug discovery. PLoS ONE, 2015; 10 (12) 0142293. doi:10.1371/journal.pone.0142293.

44. Maher MC, Lim JY, Gunawan C, Cegelski L. Cell-Based High-Throughput Screening Identifies Rifapentine as an Inhibitor of Amyloid and Biofilm Formation in Escherichia coli. Infectious Diseases. ACS Infect. Dis. 2015; 1: 460-468.

45. Chahales P, Hoffman PS, Thanassi DG. Nitazoxanide Inhibits Pilus Biogenesis by Interfering with Folding of the Usher Protein in the Outer Membrane. Antimicrob Agents Chemother. 2016 Mar 25;60(4):2028-38. doi: 10.1128/AAC.02221-15. PMID: 26824945; PMCID: PMC4808142.

46. Psonis JJ, Chahales P, Henderson NS, Rigel NW, Hoffman PS, Thanassi DG. The small molecule nitazoxanide selectively disrupts BAM-mediated folding of the outer membrane usher protein. J Biol Chem. 2019 Sep 27;294(39):14357-14369. doi: 10.1074/jbc.RA119.009616. Epub 2019 Aug 7. PMID: 31391254; PMCID: PMC6768635.

47. Brook I. Microbiology of chronic rhinosinusitis. I. Eur J Clin Microbiol Infect Dis. 2016; 35: 1059. https:// doi.org/10.1007/s10096-016-2640-x PMID: 27086363.

48. Dlugaszewska J, Leszczynska M, Lenkowski M, Tatarska A, Pastusiak T, Szyfter W. The pathophysiological role of bacterial biofilms in chronic sinusitis. Eur. Arch. Otorhinolaryngol. 2015: http://dx.doi.org/10.1007/s00405-015-3650-5.

49. Dou JL, Jiang YW, Xie JQ, Zhang XG, New is old, and old is new: recent advances in antibiotic-based, antibiotic-free and ethnomedical treatments against methicillinresistant Staphylococcus aureus wound infections. Int. J. Mol. Sci. 2016: 17. http://dx.doi.org/10.3390/ijms17050617.

50. Hassett DJ, Borchers MT, Panos RJ. Chronic obstructive pulmonary disease (COPD): evaluation from clinical, immunological and bacterial pathogenesis perspectives. J. Microbiol. 2014; 52, 211-226.

51. Scalise A, Bianchi A, Tartaglione C, Bolletta E, Pierangeli M, Torresetti M, Marazzi M, Di Benedetto G. Microenvironment and microbiology of skin wounds: the role of bacterial biofilms and related factors. Semin. Vasc. Surg. 2015; 28, 151-159.

52. Goodman SD, Obergfell KP, Jurcisek JA, Novotny LA, Downey JS, Ayala EA, Tjokro N, Li B, Justice SS, Bakaletz LO. Biofilms can be dispersed by focusing the immune system on a common family of bacterial nucleoid-associated proteins. Mucosal Immunol. 2011; 4, 625-637.

53. Mohamed, JA, Huang DB, Jiang Z, DuPont HL, Nataro JP, Belkind-Gerson J, Okhuysen PC. Association of putative enteroaggregative Escherichia coli virulence genes and biofilm production in isolates from travelers to developing countries. J. Clin. Microbiol. 2007; 45:121-126.

54. Boll EJ, Struve C, Boisen N, Olesen B, Stahlhut SG, Krogfelta KA. Role of Enteroaggregative Escherichia coli Virulence Factors in Uropathogenesis Infection and Immunity p. 2013; $1164-1171 ; 81$ (4). 10.1128/IAI.01376-12. 
55. Bolick DT, Roche JK, Hontecillas R, Bassaganya-Riera J, Nataro JP, Guerrant RL. Enteroaggregative Escherichia coli strain in a novel weaned mouse model: exacerbation by malnutrition, biofilm as a virulence factor and treatment by nitazoxanide. J Med Microbiol. 2013 Jun;62(Pt 6):896-905. doi: 10.1099/jmm.0.046300-0. Epub 2013 Mar 8. PMID: 23475903; PMCID: PMC3709553.

56. Rasko DA, Sperandio V. "Anti-virulence strategies to combat bacteria-mediated disease." Nat Rev Drug Discov 2010; 9(2): 117-128.

57. Gustave JE, Jurcisek, JA, Mccoy KS, Goodman SD, Bakaletz LO. Targeting bacterial integration host factor to disrupt biofilms associated with cystic fibrosis. J. Cyst. Fibros. 2013; 12, 384-389.

58. Brandstetter, KA, Jurcisek JA, Goodman SD, Bakaletz LO, Das S. Antibodies directed against integration host factor mediate biofilm clearance from nasopore. Laryngoscope 2013; 123, 2626-2632.

59. Novotny LA, Amer AO, Brockson ME, Goodman SD, Bakaletz LO. Structural stability of Burkholderia cenocepacia biofilms is reliant on eDNA structure and presence of a bacterial nucleic acid binding protein. PLoS One 2013; 8, e67629.

60. Brockson ME, Novotny LA, Mokrzan EM, Malhotra S, Jurcisek JA, Akbar R, Devaraj A, Goodman SD, Bakaletz LO. Evaluation of the kinetics and mechanism of action of anti-integration host factor-mediated disruption of bacterial biofilms. Mol. Microbiol. 2014; 93, 1246-1258.

61. Devaraj A, Justice SS, Bakaletz LO, Goodman SD. DNABII proteins play a central role in UPEC biofilm structure. Mol. Microbiol. 2015; 96, 1119-1135.

62. He Z, Huang Z, Zhou W, Tang Z, Ma R and Liang. Anti-biofilm Activities from Resveratrol against Fusobacterium nucleatum Frontiers in Microbiology, Front. Microbiol. 2016; 7:1065. doi: 10.3389/fmicb.2016.01065.

63. Justice SS, Li B, Downey JS, Dabdoub SM, Brockson ME, Probst GD, Ray WC, Goodman SD. Aberrant community architecture and attenuated persistence of uropathogenic Escherichia coli in the absence of individual IHF subunits. PLoS One 2012; 7, e48349.

64. Novotny LA, Jurcisek JA, Ward Jr MO, Jordan ZB, Goodman SD, Bakaletz LO. Antibodies against the majority subunit of type IV Pili disperse nontypeable Haemophilus influenzae biofilms in a LuxS-dependent manner and confer therapeutic resolution of experimental otitis media. Mol. Microbiol. 2015; 96, 276-292.

65. Hwang TJ, Dotsenko S, Jafarov A, Weyer K, Falzon D, Lunte K, Nunn P, Jaramillo E, Keshavjee S, Wares DF. Safety and availability of clofazimine in the treatment of multidrug and extensively drug-resistant tuberculosis: analysis of published guidance and meta-analysis of cohort studies. BMJ Open. 2014 Jan 2;4(1):e004143. doi: 10.1136/bmjopen-2013-004143. PMID: 24384902; PMCID: PMC3902362.

66. Cholo MC, Steel HC, Fourie PB, Germishuizen WA, Anderson R. Clofazimine: current status and future prospects. J Antimicrob Chemother. 2012 Feb;67(2):290-8. doi: 10.1093/jac/dkr444. Epub 2011 Oct 20. PMID: 22020137.

67. Yuan, S., Yin, X., Meng, X. et al. Clofazimine broadly inhibits coronaviruses including SARS-CoV-2. Nature 593, 418-423

(2021). https://doi.org/10.1038/s41586-021-03431-4

68. Sunyoto T, Potet J, Boelaert M. Why miltefosine-a life-saving drug for leishmaniasis - is unavailable to people who need it the most. BMJ Glob Health 2018;3:e000709. doi:10.1136/ bmjgh-2018-000709.

69. Tejman-Yarden N, Miyamoto Y, Leitsch D, Santini J, Debnath A, Gut J, McKerrow JH, Reed SL, Eckmann L. A reprofiled drug, auranofin, is effective against 
metronidazole-resistant Giardia lamblia. Antimicrob Agents Chemother. 2013 May;57(5):2029-35. doi: 10.1128/AAC.01675-12. Epub 2013 Feb 12. PMID: 23403423 ; PMCID: PMC3632933.

70. Ashley EA, Recht J, White NJ. Primaquine: the risks and the benefits. Malar J. 2014 Nov 3;13:418. doi: 10.1186/1475-2875-13-418. PMID: 25363455; PMCID: PMC4230503.

71. Ugboko HU, Nwinyi OC, Oranusi SU, Fatoki TH, Akinduti PA, Enibukun JM. In Silico Screening and Analysis of Broad-Spectrum Molecular Targets and Lead Compounds for Diarrhea Therapy. Bioinform Biol Insights. 2019 Oct 24;13:1177932219884297. doi: 10.1177/1177932219884297. PMID: 31695343; PMCID: PMC6820192.

72. Low JL, Wu M-L, Aziz DB, Laleu B and Dick T. Screening of TB Actives for Activity against Nontuberculous Mycobacteria Delivers High Hit Rates. Front. Microbiol. 2017, 8:1539. doi: 10.3389/fmicb.2017.01539.

73. Hennessey KM, Rogiers IC, Shih HW, Hulverson MA, Choi R, McCloskey MC, Whitman GR, Barrett LK, Merritt EA, Paredez AR, Ojo KK. Screening of the Pathogen Box for inhibitors with dual efficacy against Giardia lamblia and Cryptosporidium parvum. PLoS Negl Trop Dis. 2018 Aug 6;12(8):e0006673. doi: 10.1371/journal.pntd.0006673. PMID: 30080847; PMCID: PMC6095626.

74. Rollin-Pinheiro, R.; Borba-Santos, L.P.; da Silva Xisto, M.I.D.; de Castro-Almeida, Y.; Rochetti, V.P.; Rozental, S.; Barreto-Bergter, E. Identification of Promising Antifungal Drugs against Scedosporium and Lomentospora Species after Screening of Pathogen Box Library. J. Fungi 2021, 7, 803. https://doi.org/10.3390/jof7100803.

75. Bhargava S, Hwang J, Harris TA, George AS, Muir A, Dorff J, Okeke IN. The heat resistant agglutinin 1 is an accessory enteroaggregative Escherichia coli colonization factor.J. Bacteriol. 2009; 191: 4934-42.

76. Jenkins C, Chart H, Willshaw GA, Cheasty T, Tompkins DS. Association of putative pathogenicity genes with adherence characteristics and fimbrial genotypes in typical enteroaggregative Escherichia coli from patients with and without diarrhoea in the United Kingdom. Eur J Clin Microbiol Infect Dis 2007; 26:901-906. https://doi.org/10.1007/s10096-007-0388-z.

77. Falkow S. Molecular Koch's postulates applied to bacterial pathogenicity--a personal recollection 15 years later. Nat Rev Microbiol. 2004; 2(1):67-72. doi: 10.1038/nrmicro799. PMID: 15035010.

78. Glaubman J, Hofmann J, Bonney ME, Park S, Thomas JM, Kokona B, Ramos Falcón LI, Chung YK, Fairman R, Okeke IN. Self-association motifs in the enteroaggregative Escherichia coli heat-resistant agglutinin 1. Microbiology (Reading). 2016 Jul;162(7):1091-1102. doi: 10.1099/mic.0.000303. Epub 2016 May 10. PMID: 27166217.

79. Czeczulin JR, Balepur S, Hicks S, Phillips A, Hall R, Kothary MH, Navarro- Garcia F, Nataro JP. Aggregative adherence fimbria II, a second fimbrial antigen mediating aggregative adherence in Enteroaggregative Escherichia coli. Infect Immun. 1997; 65:4135- 4145 . 
a

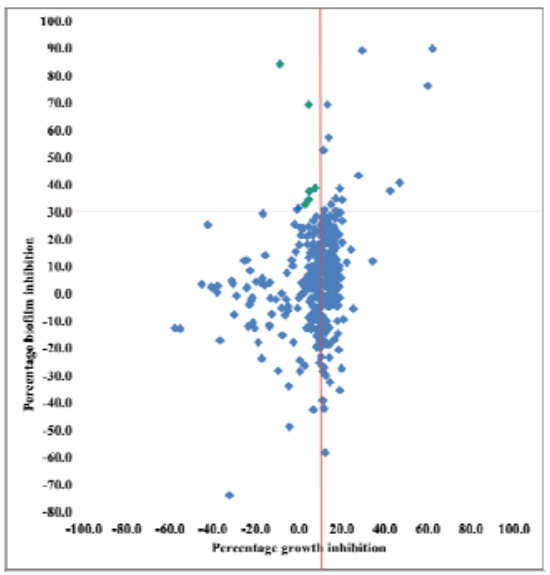

b

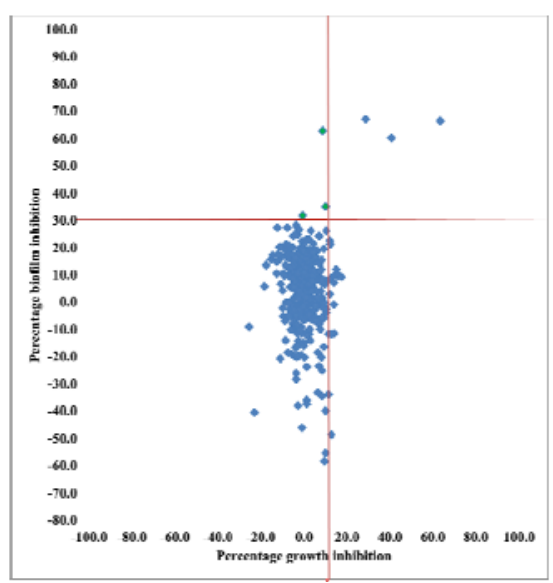

$\mathrm{c}$

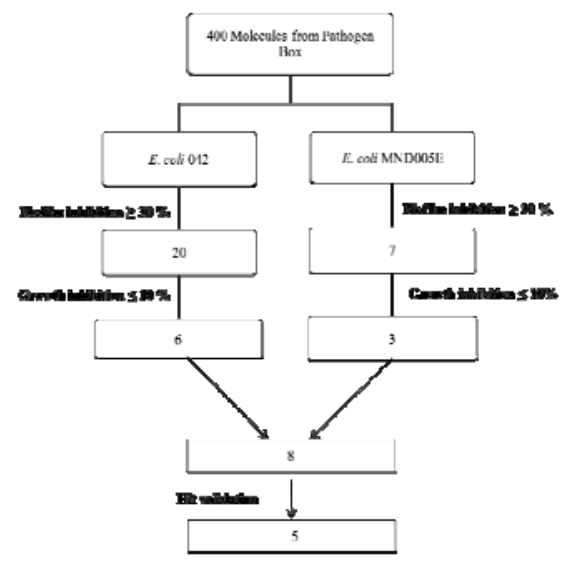

d

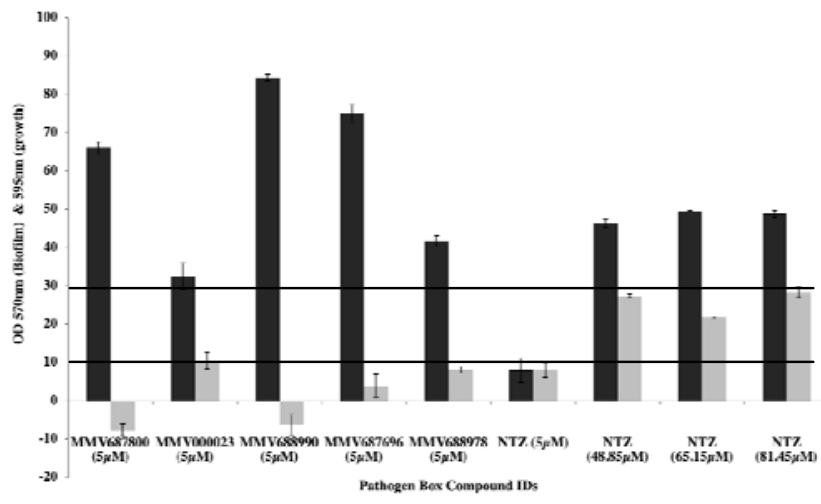

Figure 1: (a-b) Preliminary screen outcome of 400 drug-like molecules in Pathogen Box as a measure of percentage biofilm inhibition against growth inhibitions at $5 \mu \mathrm{M}$ for (a) EAEC strain 042 and (b) EAEC strain MND500E. Each compound except the antibiofilm hits is represented by a blue diamond. Compounds in the top portion of the scatterplot (above the horizontal red line) show $>30 \%$ biofilm inhibition activity and those to the left of the vertical line additionally inhibit growth by $10 \%$. The number of initial hit candidates (colored green) was six for 042 in (a) and three for MND005E in (b). (c) Hit progression cascade leading to 5 validated EAEC biofilm non growth inhibiting compounds. A total of 8 hits were obtained (One hit inhibited biofilms without inhibiting growth in both strains), but only five (validated hits) reproducibly inhibit biofilms in EAEC. (d) Biofilm inhibition (black bars) and growth inhibition (grey bars) of hit compounds and nitazoxanide against (042 and MND005E). The five hits inhibited biofilm formation by over 30\% (upper horizontal line) while inhibiting growth by under $10 \%$ (lower line). NTZ showed activity but not within the range of 
these

cut-offs.
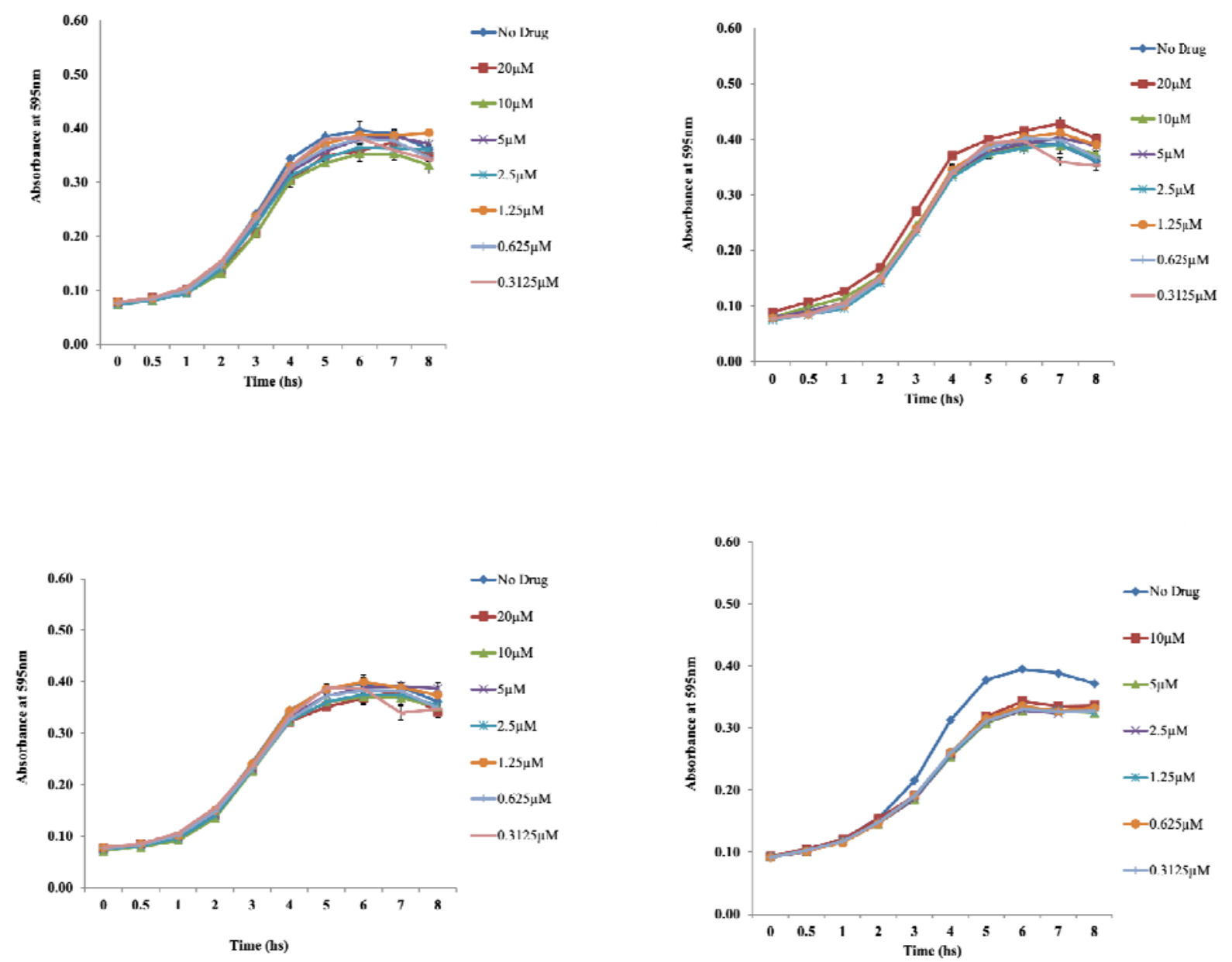

Figure 2. Hits did not significantly alter growth kinetics of EAEC strains at the various concentrations tested over 8 h: Growth kinetics of EAEC 042 with different concentrations of a) MMV688978, b) MMV687800, c) MMV688990, d) Growth kinetics of MND005E with different 
$\mathrm{a}$

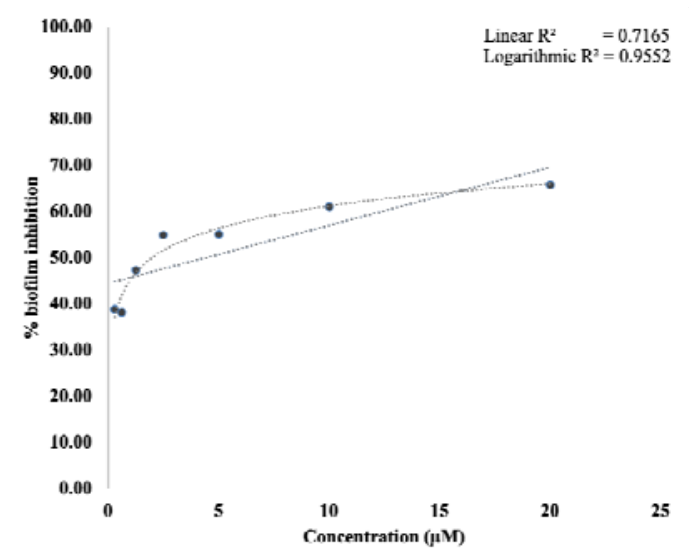

b
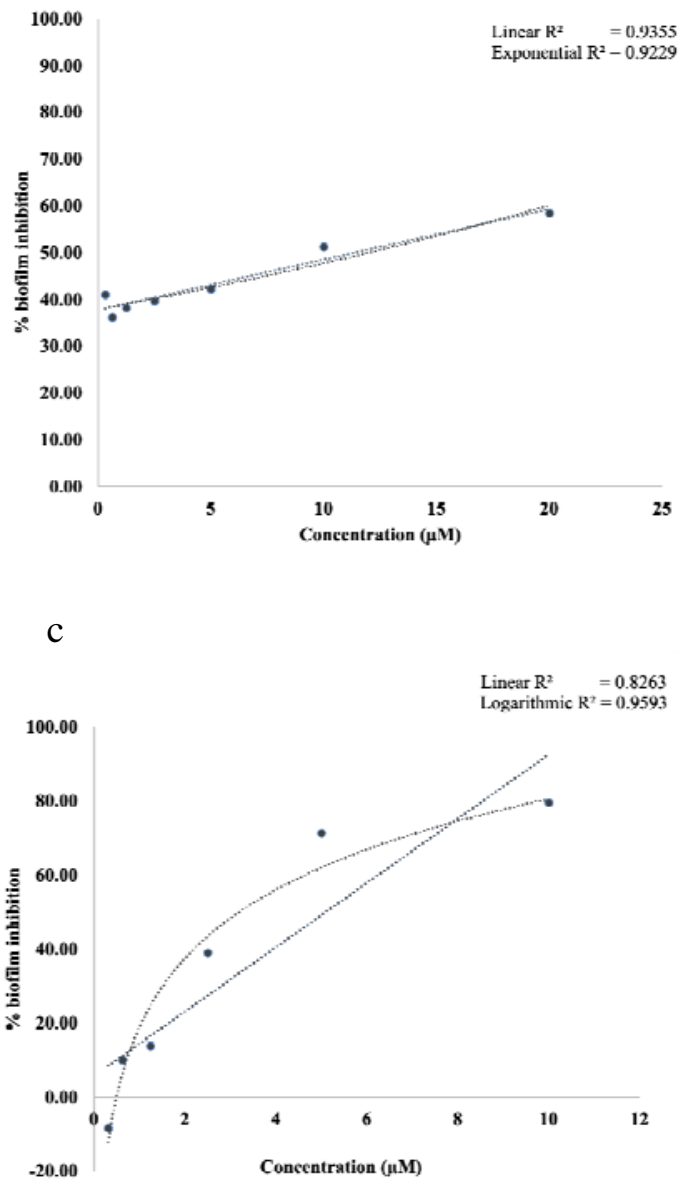

Figure 3: Regression analysis showing the relationship between percentage biofilm inhibition and drug concentrations by: a) MMV688978, (b) MMV687800, (c) MMV688990. Hits demonstrated concentration dependent biofilm inhibition and were active (meeting $>30 \%$ biofilm inhibition cut-off) at concentrations as low as $2.5 \mu \mathrm{M}$. 

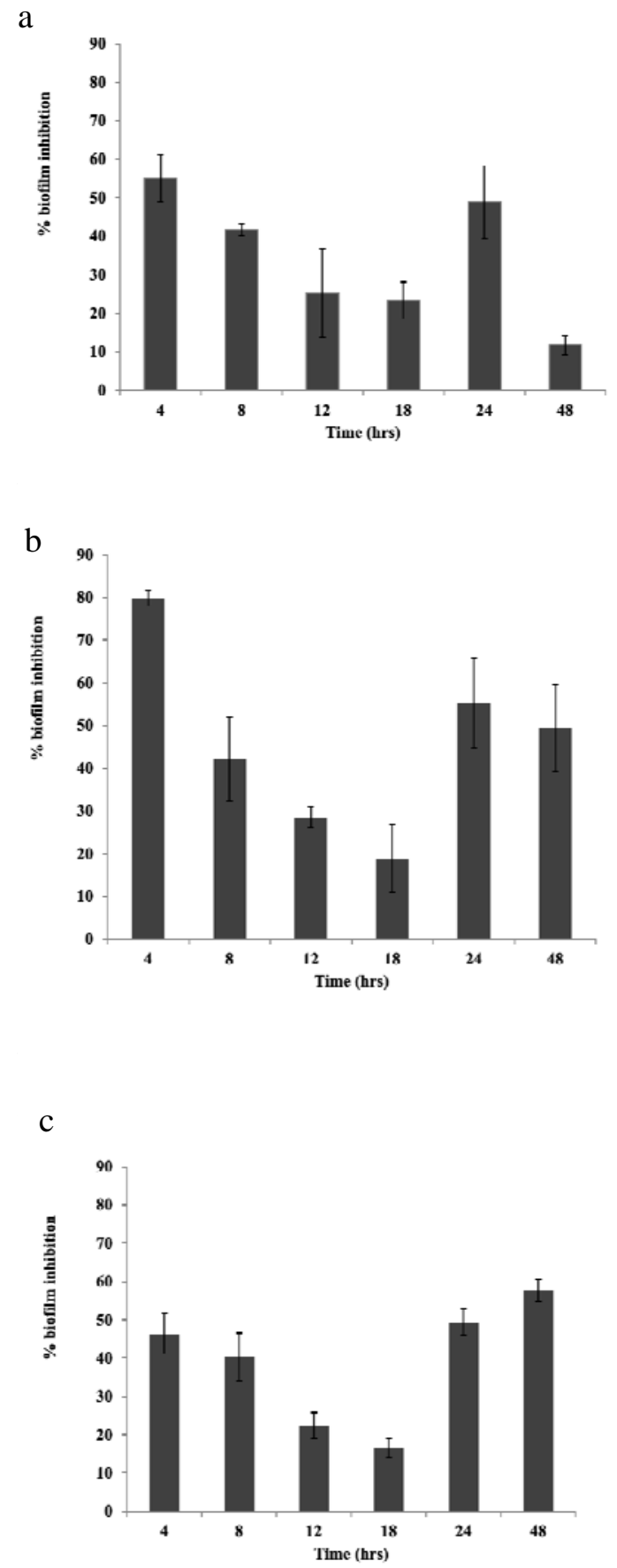

Figure 4: Time course assay of 042 biofilm (black) by 3 hits at $5 \mu \mathrm{M}$ shows inhibition is highest at the earlier time points then at later phase in: (a) MMV688978 (b) MMV687800 $\quad$ (c) MMV688990. 


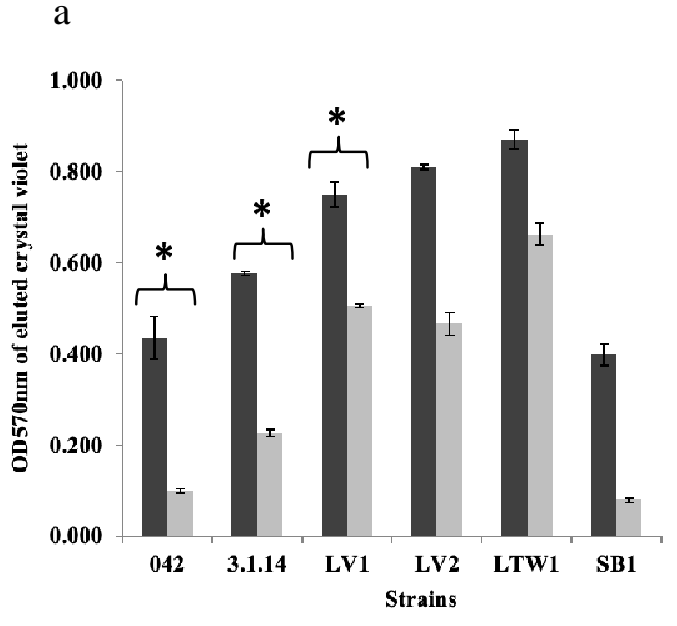

$\mathrm{c}$

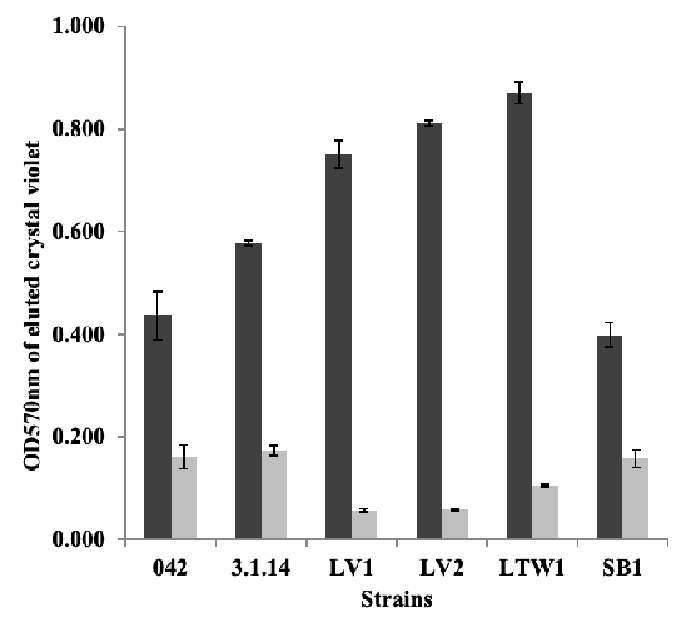

$\mathrm{C}$

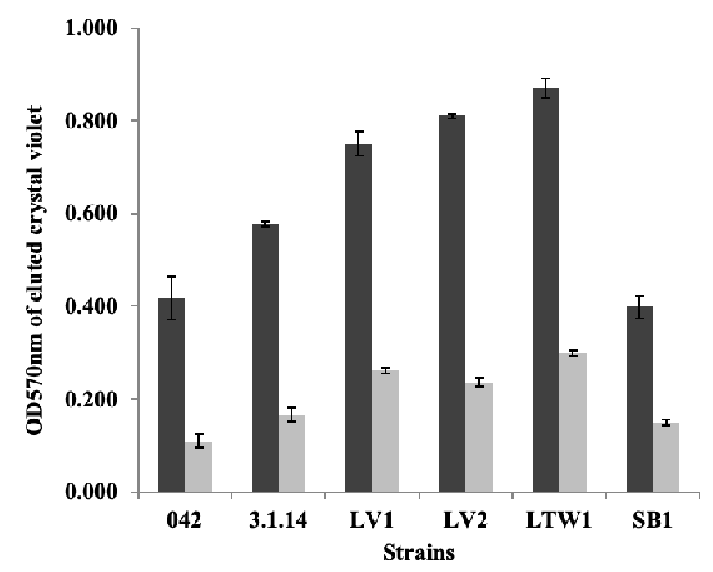

d

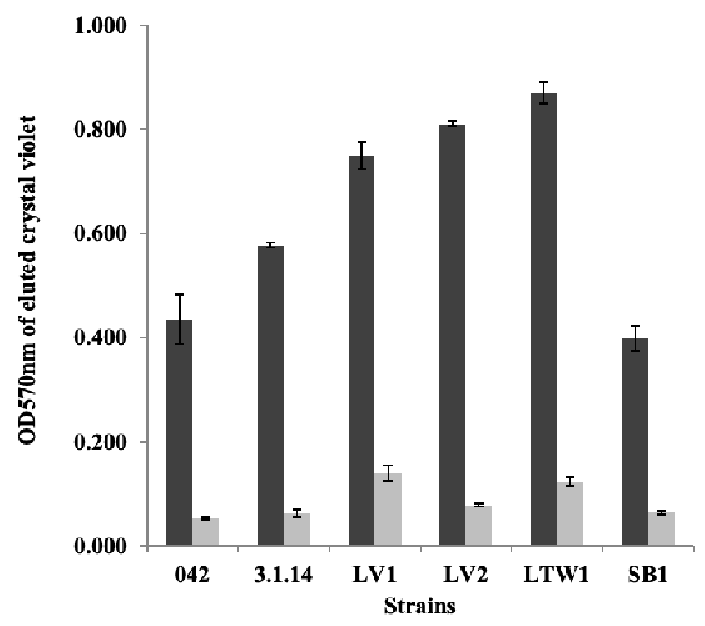

Figure 5: Effect of hits (grey) on biofilm formation (black) in E. coli 042 and 042 mutants: (a) MMV687800 (b) MMV687696 (c) MMV688978 (d) MMV688990. 042 is the prototypical EAEC strain from Peru, 3.1.14: 042 АaafA, SB1: 042 Ahra, LV1: 042 Aaap, LV2; 042 AaapAhral and LTW1: 042 $\triangle$ aap $\triangle a a f A$. Biofilm inhibition in wild type strain 042, and non-aap mutants 3.1 .14 and SB1 was highly significant $(*=\mathrm{P}<0.05)$, this was in contrast with inhibition in aap mutants LV1, LV2 and LWT1 $(\mathrm{P}>$ 0.05) indicating aap is a likely target for MMV687800. 


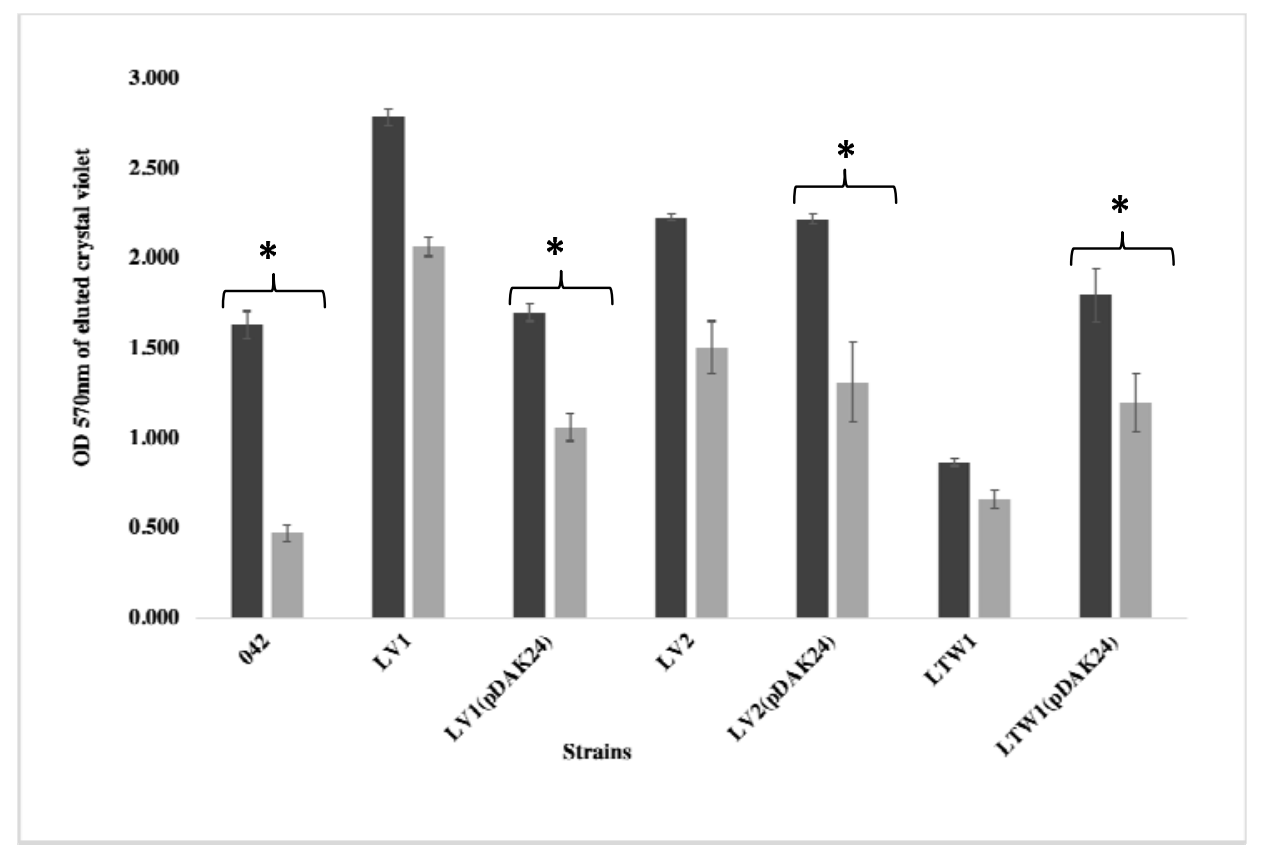

Figure 6: Biofilm inhibition of $E$. coli 042, 042 mutants and aap complimented strains by MMV687800. Biofilm formation (black) and inhibition by hit (grey). 042 is the prototypical EAEC strain

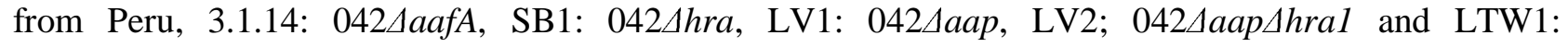
042 aap_aafA. LV1(pDAK24), LV2(pDAK24), and LTW1(pDAK24) are complement strains of LV1, LV2 and LTW1 mutants with aap inserts. Biofilm was significantly inhibited $(*=\mathrm{P}<0.05)$ in wild type strain, and in aap complemented strains compared to aap mutants ( $\mathrm{P}>0.05)$. This fulfils Molecular Koch's postulates and confirms aap's involvement in biofilm inhibition by MMV687800. 


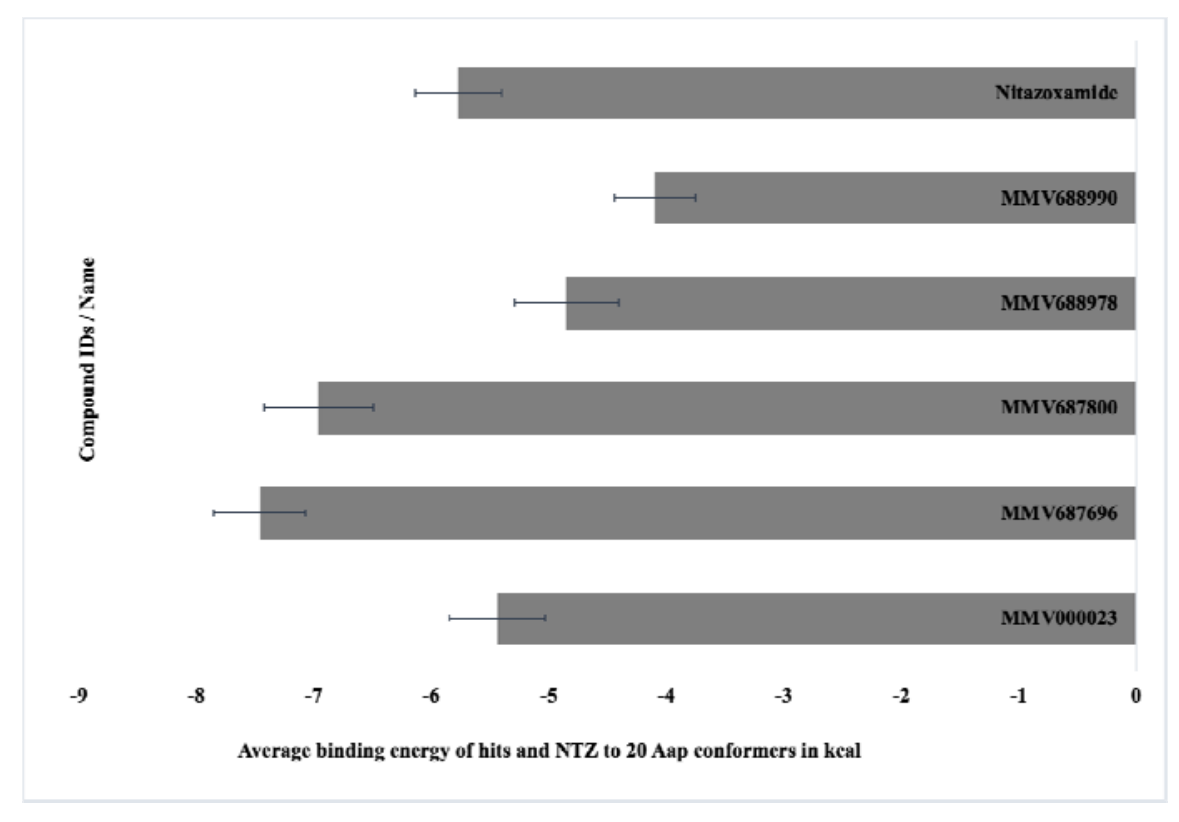

Figure 7: Average binding energy of 5 hits and NTZ to 20 conformers of Aap (kcal/mol). MMV687696 and MMV687800 demonstrated the highest binding affinity for the 20 Aap conformers. 
Table 1: Chemistry of validated hits: structures, and molecular descriptors. Data provided by MMV and PubChem

\begin{tabular}{|c|c|c|c|c|c|c|c|}
\hline Compound ID & \begin{tabular}{|l|} 
Percentage \\
blofllm \\
inhibition
\end{tabular} & $\begin{array}{l}\text { Percentage } \\
\text { growth Inhlbllon }\end{array}$ & Chemienl structure & $\begin{array}{l}\text { Molecular } \\
\text { welght }\end{array}$ & clogp & $\log D$ & \begin{tabular}{|l|} 
TPSA \\
$\left(\boldsymbol{A}^{\prime}\right)$
\end{tabular} \\
\hline MMV687800 & 66.23 & -7.63 & a & 473.40 & 6.28 & 5.44 & 40.00 \\
\hline \begin{tabular}{|l|} 
MMV688978 \\
\end{tabular} & 41.82 & 8.20 & & 678.48 & 1.40 & - & 115.00 \\
\hline MMV000023 & 32.65 & 10.47 & & 455.35 & 1.90 & 3.19 & \begin{tabular}{|l|l|}
60.20 \\
\end{tabular} \\
\hline MMV688990 & 84.40 & \begin{tabular}{|c|c|}
-6.21 \\
\end{tabular} & & 407.57 & 0.12 & 5.82 & \begin{tabular}{|l|l|}
58.60 \\
\end{tabular} \\
\hline MMV687696 & 75.23 & 4.04 & & 557.01 & 6.61 & 5.70 & - \\
\hline $\begin{array}{l}\text { MMV688991 } \\
\text { (NTZ) }\end{array}$ & 8.10 & 8.20 & & 307.28 & 1.63 & - & 142.00 \\
\hline
\end{tabular}


Table 2: Reference strains and EAEC strains used in this screen

\begin{tabular}{|c|c|c|}
\hline EAEC Strains or plasmids & Description or genotype & $\begin{array}{l}\text { Reference/ } \\
\text { source }\end{array}$ \\
\hline \multicolumn{3}{|l|}{ Strains } \\
\hline 042 & Prototypical genome-sequenced EAEC isolate originally from Peru & (33) \\
\hline $60 \mathrm{~A}$ & $\begin{array}{l}\text { EAEC isolate from Mexico, which does not express AAF/II or Hra1 but harbour genes } \\
\text { encoding AAF/I and Hra2 }\end{array}$ & $(41)$ \\
\hline ER2523 & $\begin{array}{l}\text { E. coli B derivative }(\text { fhuA2 [lon] ompT gal sulA11 R(mcr- 73::miniTn10--TetS)2 [dcm] } \\
R(z g b-210:: T n 10--T e t S) \text { endA1 } \Delta(\text { mcrC-mrr }) 114: \because I S 10)\end{array}$ & $(\mathrm{NEB})$ \\
\hline LV1(pDAK24) & LV1 strain carrying aap gene cloned into pMal-c5X (pDAK24) & This study \\
\hline LV2(pDAK24) & LV2 strain carrying aap gene cloned into pMAL-c5X (pDAK24) & This study \\
\hline LTW1(pDAK24) & LTW1 strain carrying aap gene cloned into pMAL-c5X (pDAK24) & This study \\
\hline $\begin{array}{l}\text { CHD076J, CHD61D, JKD76I, LKD69F, } \\
\text { LKD69G, LKD71D, LLD106E, LLD28J, } \\
\text { LLD33B, LLD52A, LLD53H, LLD89B, } \\
\text { LLD89E, LLD9B, LWD45C, LWD45D, } \\
\text { MND005E, MND081E, MND57C, } \\
\text { MND58E, MND58I, MND60A, MND60D, } \\
\text { MND60E, MND61B }\end{array}$ & $\begin{array}{l}\text { EAEC strains isolated from children with diarrhoea and characterised in our lab in } \\
\text { Nigeria }\end{array}$ & $(18)$ \\
\hline \multicolumn{3}{|l|}{ Mutants } \\
\hline 3.1 .14 & 042 with inactivating TnphoA insertion in aafA & $(79)$ \\
\hline SB1 & $042 \Delta h r a$, isogenic mutant; hral::aphA-3 & $(73)$ \\
\hline LV1 & 042 $4 a a p$, isogenic mutant. aap:: dhfrA7 & $(40)$ \\
\hline LV2 & 042Aaap $\Delta h r a$, isogenic mutant, aap:: dhfrA7, hral::aphA-3 & $(40)$ \\
\hline LWT1 & 042_aap_aafA, isogenic mutant, aap:: dhfrA7, aafA::TnphoA & $(40)$ \\
\hline \multicolumn{3}{|l|}{ Plasmids } \\
\hline pMAL-c5X & Bacterial vector with inducible maltose-binding protein (MBP) fusions in cytoplasm & $(\mathrm{NEB})$ \\
\hline pDAK24 & aap from 042 cloned into $S b f I$ and $M f e I$ sites of pMAL-c5X & (This study) \\
\hline
\end{tabular}


Table 3 : EAEC strains and antibiofilm activity spectra profile of 5 hit

\begin{tabular}{cccccc}
\hline \multirow{2}{*}{ Strains } & \multicolumn{5}{c}{ Hits and percentage biofilm inhibition } \\
\cline { 2 - 6 } & MMV687800 & MMV68890 & MMV688978 & MMV678696 & MMV000023 \\
\hline 042 & 76.52 & 69.87 & 60.70 & 47.19 & 7.20 \\
60A & 35.60 & 59.31 & 39.02 & 38.73 & 61.25 \\
CHD076J & 5.85 & -18.46 & -12.62 & 13.69 & 0.31 \\
CHD61D & 18.94 & 12.52 & 24.28 & -3.88 & 3.79 \\
JKD76I & 45.82 & 12.50 & 25.15 & 4.85 & 20.22 \\
LKD69F & -21.59 & 1.06 & 27.12 & -15.50 & -48.34 \\
LKD69G & 6.23 & -9.86 & 12.46 & -16.26 & -69.27 \\
LKD71D & -6.15 & 13.20 & 3.39 & -53.39 & -3.47 \\
LLD106E & 77.28 & 38.06 & 47.08 & 45.06 & 35.01 \\
LLD28J & 52.02 & 51.27 & 59.80 & 15.54 & 58.09 \\
LLD33B & -10.88 & -5.88 & 1.47 & -19.12 & -16.76 \\
LLD52A & -50.74 & -19.37 & 18.53 & -32.56 & -46.46 \\
LLD53H & 44.15 & 19.84 & 48.12 & 43.43 & 37.09 \\
LLD89B & 11.64 & 57.28 & 39.84 & 33.27 & -20.39 \\
LLD89E & 1.69 & 6.26 & 36.15 & -14.61 & 32.00 \\
LLD9B & 7.68 & -15.45 & 4.25 & -11.47 & -3.89 \\
LWD45C & 60.86 & -17.73 & -13.03 & -11.99 & -12.38 \\
LWD45D & 45.19 & -28.02 & -16.80 & -15.18 & -15.75 \\
MND005E & -40.17 & 62.70 & 5.19 & -58.71 & 35.61 \\
MND081E & -1.39 & -14.49 & 17.96 & -35.00 & -33.99 \\
MND57C & 0.41 & -11.59 & 3.19 & -26.96 & -33.15 \\
MND58E & -11.59 & -21.77 & 2.96 & -1.35 & -11.59 \\
MND58I & -4.84 & -22.13 & -4.72 & -13.71 & -12.10 \\
MND60A & -50.80 & -9.83 & 9.48 & -32.47 & -40.95 \\
MND60D & -72.48 & -5.87 & 4.91 & -20.64 & -57.25 \\
MND60E & 36.00 & 15.89 & -2.02 & -13.00 & -47.09 \\
MND61B & -4.91 & -15.74 & 25.70 & -10.75 & -28.04 \\
\hline
\end{tabular}


Table 4: Free energy of interaction between hit molecules and AAF/II major and minor subunits of EAEC strain 042.

Hits / NTZ

AAF/II major subunit binding free AAF/II minor subunit binding free energy (kcal/mol) energy (kcal/mol)

\begin{tabular}{lllll}
\hline & Blind docking & Focused docking & Blind docking & Focused docking \\
\hline MMV000023 & -5.6 & -5.5 & -6.7 & -4.7 \\
MMV687696 & -8.6 & -3.8 & -9.6 & -4.1 \\
MMV687800 & -6.9 & -5.7 & -8.8 & -4.4 \\
MMV688978 & -5.0 & -5.2 & -6.3 & -4.2 \\
MMV688990 & -4.7 & -4.5 & -5.0 & -3.9 \\
Nitazoxanide & -5.4 & -6.5 & -6.2 & -5.5 \\
\hline
\end{tabular}


Table S1: Classification of pathogen box compounds matched with biofilm inhibition screen outcome

\begin{tabular}{lllll}
\hline $\begin{array}{l}\text { Pathogen box } \\
\text { compound class }\end{array}$ & $\begin{array}{l}\text { Number of } \\
\text { compounds }\end{array}$ & $\begin{array}{l}\text { Antibiofilms } \\
\text { in this } \\
\text { screen }\end{array}$ & $\begin{array}{l}\text { Antibacterials } \\
\text { in this screen }\end{array}$ & $\begin{array}{l}\text { Unvalidated } \\
\text { antibiofilm agents }\end{array}$ \\
\hline Tuberculosis & 116 & 1 & 0 & 0 \\
Malaria & 125 & 0 & 1 & 0 \\
Kinetoplastids & 70 & 0 & 2 & 3 \\
Helminths & 32 & 0 & 1 & 0 \\
Crytosporidiosis & 11 & 0 & 1 & 0 \\
Toxoplasmosis & 15 & 0 & 0 & 0 \\
Dengue & 5 & 0 & 0 & 0 \\
Reference Compounds & 26 & 4 & 4 & 0 \\
Total & $\mathbf{4 0 0}$ & $\mathbf{5}$ & $\mathbf{9}$ & $\mathbf{3}$ \\
\hline
\end{tabular}



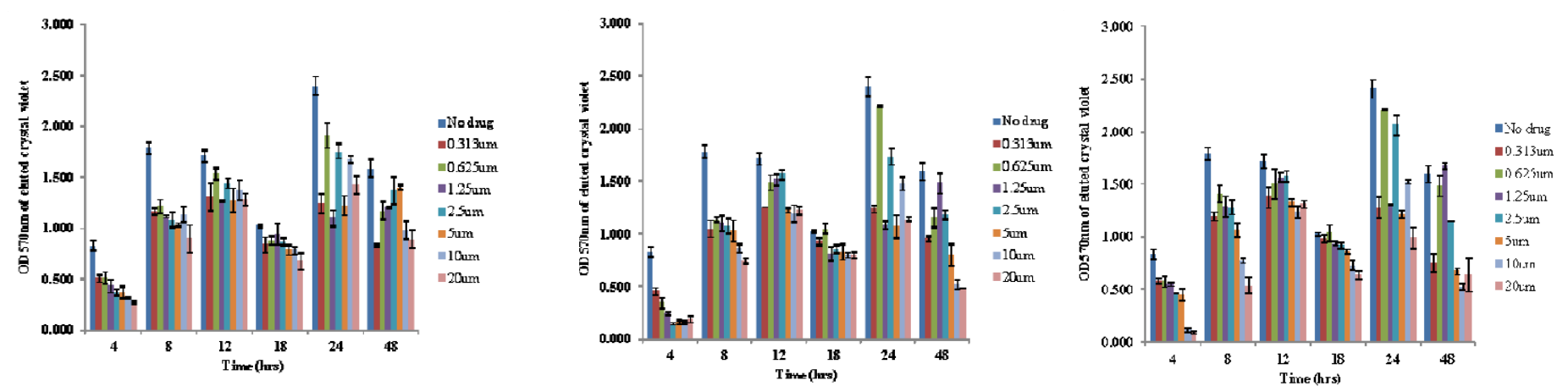

Figure S1: Time course inhibition of biofilms in EAEC 042 using different hit concentrations of (a) MMV688978, (b) MMV687800, (c) MMV688990. 